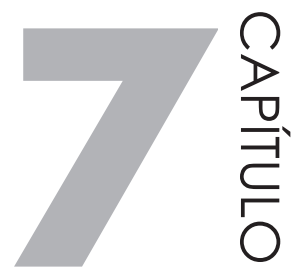

\title{
RELATIVIDADE ESPECIAL OU RESTRITA
}

\subsection{INTRODUÇÃO}

No século XIX, fazia-se uma analogia entre ondas luminosas e ondas sonoras ou outro tipo de perturbação puramente mecânica. Postulava-se a existência de um "éter", "éter luminífero" ou "portador de luz”, substância tênue que ocuparia todo o espaço e serviria como meio de propagação da luz.

Em 1881, Albert Abraham Michelson incumbiu-se da tarefa de submeter o éter, caso existisse, a uma verificação física direta. Michelson, mais tarde auxiliado por Edward Williams Morley tentavam determinar a velocidade com que a Terra se movia através do éter. O interferômetro de Michelson foi escolhido para essa experiência, conhecida agora como a famosa experiência de Michelson-Morley (ver Seção 2.1, sobre medição do comprimento de onda da luz).

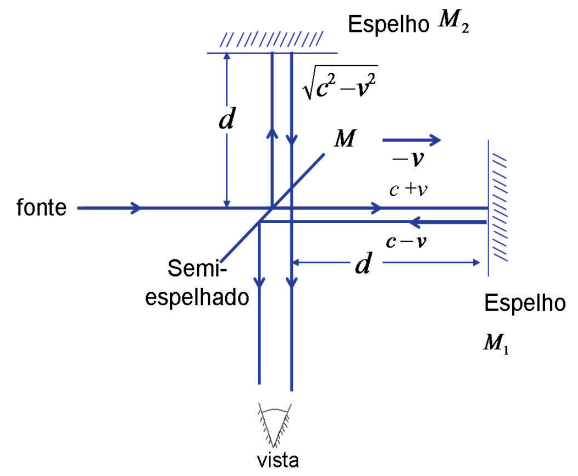


A Terra se desloca junto com o interferômetro com uma velocidade $v$ através do éter, ou seja, o éter passa pelo interferômetro com velocidade $-v$.

De $M$ a $M_{1}$ a velocidade será $c+v$.

De $M_{1}$ a $M$ a velocidade será $c-v$.

Pelo teorema de Pitágoras, obtemos a velocidade em $M_{2} M: \sqrt{c^{2}-v^{2}}$. A seguir gira-se o interferômetro em $90^{\circ}$ e efetuam-se novas medições.

Para grande surpresa de Michelson e Morley, a velocidade verificada era a mesma em todas as direções.

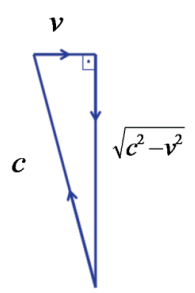

O enigma foi resolvido em 1905, quando Einstein admitiu a velocidade da luz no vácuo como um invariante físico, tendo o mesmo valor para todos os observadores.

A definição de evento é uma ocorrência para a qual um observador pode associar três coordenadas espaciais e uma temporal.

Postulado da relatividade especial ou restrita: a velocidade da luz no vácuo tem o mesmo valor $c$ em todas as direções e em todos os referenciais inerciais, isto é, as velocidades entre si são uniformes, não estão aceleradas.

A velocidade da luz é uma velocidade limite. Isso foi demonstrado em uma experiência por W. Bertozzi em 1964. Ele acelerou elétrons a diversas velocidades. À medida que a força atua sobre um elétron muito rápido, sua energia cinética também aumenta, tendendo para valores muito grandes, porém, sua velocidade não aumenta consideravelmente. Os elétrons foram acelerados até pelo menos 0,999.999.999.95c. Embora muito próxima de $c$, esta velocidade ainda é menor que $c$.

Sobre o postulado da invariância da velocidade da luz foi feita uma experiência no CERN ${ }^{1}$ em 1964, usando "píons neutros", $\pi^{0}$, em um acelerador de partículas. Elas decaem em dois raios $\gamma$, que fazem parte do espectro eletromagnético: $\pi^{0} \rightarrow \gamma+\gamma$.

Conseil Européen pour la Recherche Nucléaire, em Meyrim, fronteira franco-suíça, com um síncroton de prótons de $28 \mathrm{GeV}$ e outro de $450 \mathrm{eV}$, desde 1976 . 
O feixe foi acelerado até $0,99975 c$ e mediu-se a velocidade dos raios $\gamma$.

Os resultados obtidos foram:

- Luz dos píons em movimento:

$$
2,998 \times 10^{8} \mathrm{~m} / \mathrm{s}
$$

- Luz de uma fonte em repouso (valor aceito de $c$ ): $2.998 \times 10^{8} \mathrm{~m} / \mathrm{s}$.

Píons são mésons $\pi$, partículas originárias de raios cósmicos.

A velocidade da luz emitida pelos píons, que se deslocavam com velocidade quase igual à da luz, era a mesma que seria medida se os píons estivessem em repouso no laboratório.

\subsection{RELATIVIDADE DO TEMPO}

Seja um observador posicionado dentro de um trem com velocidade uniforme $v$ em relação a uma estação. No vagão, ocorre um evento 1 , em que se emite do chão um sinal luminoso, refletido por um espelho no teto para o chão do vagão, onde é recebido no evento 2. A distância do chão ao teto é $\mathrm{D}$. $\mathrm{O}$ intervalo de tempo entre os dois eventos é $\Delta t_{0}$. Para o observador, os eventos e o intervalo de tempo estão em repouso, pois ocorrem dentro vagão.

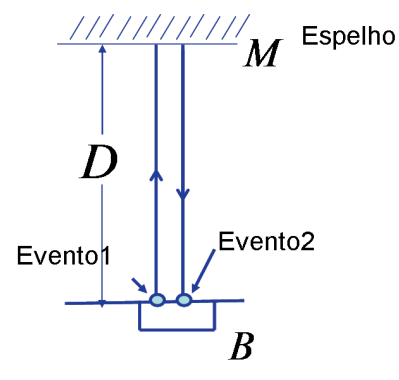

Esse intervalo de tempo é denominado "tempo próprio". Teremos: $\Delta t_{0}=\frac{2 D}{c}$

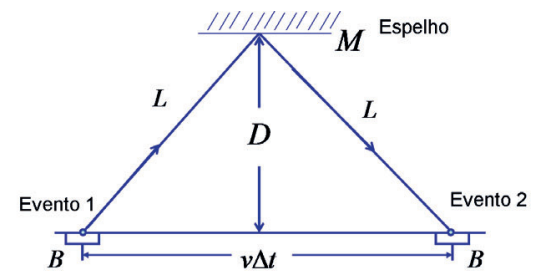

Outro observador, posicionado na plataforma, observa o trem passar. Como a velocidade da luz é a mesma para os dois observadores, o intervalo de tempo entre os dois eventos, para o observador na 
plataforma, que observa o trem em movimento uniforme com velocidade $v$, será: $\Delta t=\frac{2 L}{c} \quad$ em que,

$$
\begin{aligned}
& L=\sqrt{(1 / 2 v \Delta t)^{2}+D^{2}}=\sqrt{(1 / 2 v \Delta t)^{2}+\left(1 / 2 c \Delta t_{0}\right)^{2}}=c \frac{\Delta t}{2} \Rightarrow \mathrm{v}^{2} \Delta \mathrm{t}^{2}+\mathrm{c}^{2} \Delta \mathrm{t}_{0}^{2}=\mathrm{c}^{2} \Delta \mathrm{t}^{2} \Rightarrow \\
& \Rightarrow \Delta \mathrm{t}^{2}\left(\mathrm{c}^{2}-\mathrm{v}^{2}\right)=\mathrm{c}^{2} \Delta \mathrm{t}_{0}^{2} \Rightarrow \Delta \mathrm{t}^{2}=\frac{\Delta \mathrm{t}_{0}^{2}}{\left(\mathrm{c}^{2}-\mathrm{v}^{2}\right) / \mathrm{c}^{2}}
\end{aligned}
$$

Resulta: $\Delta t=\frac{\Delta t_{0}}{\sqrt{1-v^{2} / c^{2}}}$, ver demonstração mais atualizada no final do Anexo 3, secção A3.2 em:

"Relatividade do tempo".

À relação $\beta=\frac{v}{c}$ chamamos "parâmetro de velocidade" e à expressão $\gamma=\frac{1}{\sqrt{1-\beta^{2}}}(0)^{2}$ chamamos de "fator de Lorentz", pois Hendrik Lorentz, físico holandês, em 1890, foi o primeiro a obter a expressão, em conexão com o problema do campo eletromagnético de uma carga em movimento. No entanto, tanto ele como o físico irlandês George Fitzgerald apresentou a hipótese de que os objetos sólidos se contraíam ligeiramente quando se moviam no éter. Essa contração reduziria tudo, inclusive os braços medidores do interferômetro de Michelson-Morley, na medida exata necessária para tornar o efeito do éter na luz impossível de identificar. Porém, a hipótese de Einstein, que vimos na introdução, comprovou ser a verdadeira.

Suponhamos um primeiro evento consistindo na emissão de um sinal que se propaga com a velocidade da luz, partindo de um ponto de coordenadas $x_{1}, y_{1}, z_{1}$, no instante $t_{1}$. Um segundo evento consistirá na chegada do sinal ao ponto $x_{2}, y_{2}, z_{2}$, no instante $t_{2}$. O sinal se propaga com a velocidade $c$ e o caminho percorrido é $c\left(t_{2}-t_{1}\right)$.

Então teremos: $c\left(t_{2}-t_{1}\right)=\left[\left(x_{2}-x_{1}\right)^{2}+\left(y_{2}-y_{1}\right)^{2}+\left(z_{2}-z_{1}\right)^{2}\right]^{1 / 2}$

Quando $x_{1}, y_{1}, z_{1}, t_{1}$ e $x_{2}, y_{2}, z_{2}, t_{2}$ forem as coordenadas de dois eventos quaisquer, a quantidade:

$$
s_{12}=\left[c^{2}\left(t_{2}-t_{1}\right)^{2}-\left(x_{2}-x_{1}\right)^{2}-\left(y_{2}-y_{1}\right)^{2}-\left(z_{2}-z_{1}\right)^{2}\right]^{1 / 2}
$$

Será chamada de "intervalo" entre os dois eventos.

Se dois eventos forem infinitamente vizinhos, seu intervalo $d s$ se escreverá:

$$
d s^{2}=c^{2} d t^{2}-d x^{2}-d y^{2}-d z^{2}(1)
$$

\footnotetext{
2 Chamada contração de Lorentz-Fitzgerald.
} 
Seja um intervalo de tempo $d t$ durante o qual se percorre a distância $\sqrt{d x^{2}+d y^{2}+d z^{2}}$. O intervalo $d t$ é indicado por um relógio em repouso. Qual será o tempo $d t^{\prime}$ indicado por um relógio que se move? No sistema de coordenadas ligado ao relógio móvel, este estará em repouso, ou seja, $d x^{\prime}=d y^{\prime}=d z^{\prime}=0$. Como o intervalo entre dois eventos é invariante, temos:

$d s^{2}=c^{2} d t^{2}-d x^{2}-d y^{2}-d z^{2}=c^{2} d t^{\prime 2}$ (ver Análise dos intervalos de eventos $d s$ Seção 7.3, Equação $1)$.

Portanto: $d t^{\prime}=\frac{d s}{c}=\frac{1}{c} \sqrt{c^{2} d t^{2}-d x^{2}-d y^{2}-d z^{2}}$

Ou seja: $d t^{\prime}=d t \sqrt{1-\frac{d x^{2}+d y^{2}+d z^{2}}{c^{2} d t^{2}}}=\mathrm{dt} \sqrt{\frac{\mathrm{c}^{2} \mathrm{dt}^{2}-\mathrm{dx}^{2}-\mathrm{dy}^{2}-\mathrm{dz} \mathrm{z}^{2}}{\mathrm{c}^{2} \mathrm{dt}^{2}}}$

Como: $\frac{d x^{2}+d y^{2}+d z^{2}}{d t^{2}}=v^{2} \quad v$ é a velocidade do relógio móvel

Logo: $d t^{\prime}=\frac{d s}{c}=d t \sqrt{1-\frac{v^{2}}{c^{2}}}$

Integrando, obtemos:

$$
t_{2}^{\prime}-t_{1}^{\prime}=\int_{t_{2}}^{t_{1}} d t \sqrt{1-\frac{v^{2}}{c^{2}}}
$$

Esse intervalo de tempo é chamado de tempo próprio.

Voltando, teremos: $\Delta t=\gamma \Delta t_{0}$.

Como $\gamma \geq 1$, teremos: $T_{\text {movimento }} \geq T_{\text {repouso }}$

Isto é, quando em movimento, o tempo transcorre mais lentamente, ou seja, há uma dilatação do tempo.

Prova: por testes em laboratório sabemos que a vida média dos múons é de $2,2 \mu s$, portanto $\Delta t_{0}=2,2 \mu s$ (em repouso).

Realizou-se uma experiência em 1977 no CERN com um feixe de múons acelerados em um anel de $7,0 \mathrm{~m}$ de raio até atingir a velocidade de $v=0,9994 \mathrm{c}$. Obteve-se $\gamma=28,87$, portanto $\Delta t=\gamma \Delta t_{0}$ $\Delta t=63,5 \mu \mathrm{s}$. Os múons são mésons $\mu$, partículas originárias de raios cósmicos.

$\mathrm{O}$ valor medido coincidiu, dentro dos erros experimentais com $\Delta t$ calculado.

${ }^{3}$ Se $v$ for constante, como é o caso, obtemos: $\Delta t^{\prime}=\Delta t / \gamma$ e $\therefore \Delta t=\gamma \Delta t_{0}$ como a Equação (0). 
Para uma demonstração mais elegante e atualizada da relatividade do tempo, ver Anexo 3 secção A3.2, em: Relatividade do tempo.

\subsubsection{0 decaimento do múon}

Para esclarecer o assunto apresentado anteriormente, convém desenvolver o seguinte: o múon se forma no topo da atmosfera, a cerca de $9 \mathrm{~km}$ de altitude, quando essa camada é atingida por vários raios cósmicos (partículas altamente energéticas do espaço exterior). Em repouso, os múons se desintegram em $2,2 \mu \mathrm{s}(0,0000022 \mathrm{~s})$. Ao serem formados, apresentam velocidades muito próximas da luz, cerca de $0,998 \mathrm{c}$.

Distância percorrida na atmosfera até desintegrar:

$$
d_{m}=v \cdot t \Rightarrow d_{m}=2,994 \cdot 10^{8} \mathrm{~m} / \mathrm{s} \cdot 2,2 \cdot 10^{-6} \mathrm{~s} \Rightarrow d_{m}=650 m
$$

Se são gerados a $9 \mathrm{~km}$ e percorreriam cerca de $650 \mathrm{~m}$, como podem ser observados na superfície da Terra? Devido à alta velocidade, sofrem efeito relativístico, causando uma dilatação do tempo, a qual é fornecida pela equação que leva em conta o fator de Lorentz:

$$
\begin{aligned}
& \Delta \mathrm{t}_{\mathrm{a}}=\frac{\Delta \mathrm{t}_{\mathrm{m}}}{\sqrt{1-\frac{\mathrm{v}^{2}}{\mathrm{c}^{2}}}} \Rightarrow \Delta \mathrm{t}_{\mathrm{a}}=\frac{\Delta \mathrm{t}_{\mathrm{m}}}{\sqrt{1-\frac{(0,998)^{2} \mathrm{c}^{2}}{\mathrm{c}^{2}}}} \Rightarrow \Delta \mathrm{t}_{\mathrm{a}}=\frac{2,2 \cdot 10^{-6}}{0,063} \\
& \therefore \Delta \mathrm{t}_{\mathrm{a}}=3,5 \times 10^{-6} \mathrm{~s}
\end{aligned}
$$

No referencial do solo, a distância percorrida pelo múon antes de se desintegrar é:

$$
\begin{gathered}
\mathrm{d}_{\mathrm{a}}=\mathrm{v} \cdot \Delta \mathrm{t}_{\mathrm{a}} \Rightarrow \mathrm{d}_{\mathrm{a}}=2,994 \cdot 10^{8} \mathrm{~m} / \mathrm{s} \cdot 3,5 \cdot 10^{-6} \mathrm{~s} \\
\therefore \mathrm{d}_{\mathrm{a}} \cong 10.000 \mathrm{~m}
\end{gathered}
$$

Por causa da dilatação do tempo, o múon consegue atingir a superfície da Terra e ser detectado.

\subsubsection{0 tempo e os buracos negros}

Quando uma estrela de massa grande ${ }^{4}$ esgota seu combustível nuclear, perde calor e se contrai. A deformação do espaço-tempo nessa estrela é tão grande que causa a criação de um buraco negro do qual nem a luz consegue escapar e cujo tempo chegará ao fim.

${ }^{4}$ Chandrasekhar, astrofísico norte-americano de origem hindu, prêmio Nobel de física em 1983, demonstrou que as estrelas com massa de até 1,44 do Sol, transformam-se em anãs brancas quando colapsam ao final do processo de conversão do $H$ em He. Acima desse limite (chamado de "limite de Chandrasekhar") o colapso resulta em estrelas de nêutrons ou, quando forem supermassivas, em buracos negros. Conforme HAWKING em "O Universo em uma casca de noz", sabemos agora que não há configurações de estado final para estrelas com mais de duas vezes a massa do sol. Essas estrelas continuarão encolhendo até se tornarem buracos negros, regiões do espaço-tempo tão distorcidas que a luz não consegue escapar delas. 


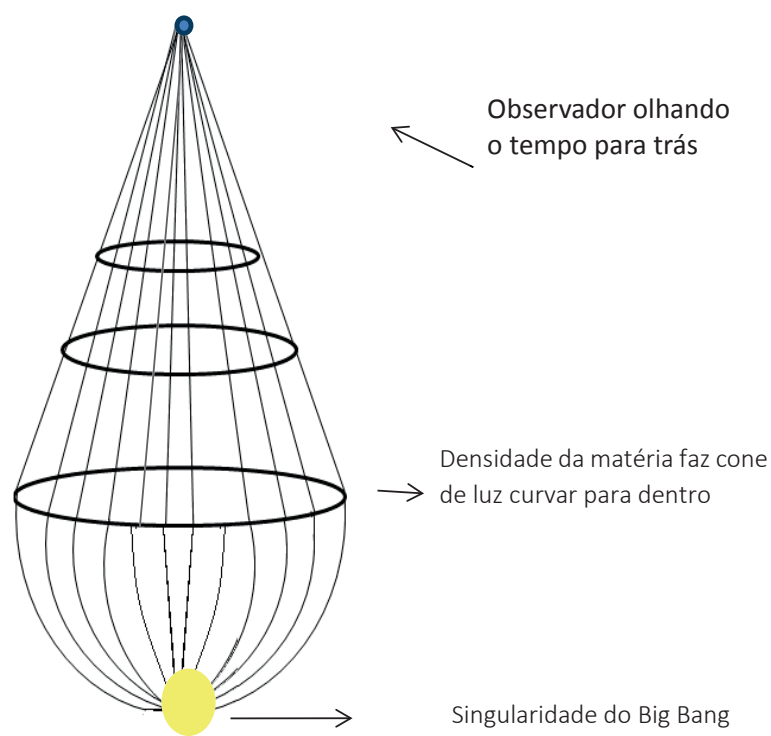

O tempo tem forma de pera.

Inicialmente, no Big Bang, a densidade da matéria seria infinita.

\subsubsection{Analogia com os meridianos}

Os meridianos se iniciam em um polo e terminam no outro. Cada meridiano tem uma determinada hora. Nos polos o tempo fica parado. É o que ocorre nos Buracos negros (HAWKING em "O Universo em uma casca de noz").

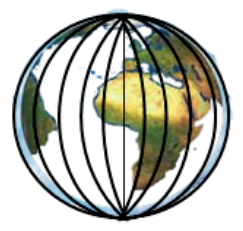

Isso será mais bem explicado no segundo volume desta obra, sobre a Relatividade Geral. 
Se compararmos com os paralelos de latitude (conforme a Figura), considerando cada latitude como $\Delta t$, isto é, variação de tempo, e supondo que a medida que as latitudes se aproximem dos polos, tendam para zero, ou seja, $\Delta t \rightarrow 0$. Quando atingir o polo, haverá $\Delta t=0$, isto é, o tempo para.

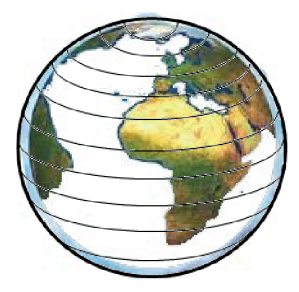

\subsection{RELATIVIDADE DO COMPRIMENTO}

Um observador em repouso observa um trem em movimento e mede o comprimento $L$ do trem, durante um intervalo de tempo $\Delta t_{0}$, medido em repouso, logo: $L=v \Delta t_{0}$.

Outro observador dentro do trem mede o comprimento do trem $L_{0}$, que, para esse observador está em repouso em relação a ele. Esse observador constata que o tempo para o trem passar pela plataforma foi de $\Delta t$, sendo que esse intervalo foi medido em relação à plataforma que para esse observador estava em movimento, logo: $L_{0}=v \Delta t$.

Dividindo as duas expressões entre si: $\frac{L}{L_{0}}=\frac{v \Delta t_{0}}{v \Delta t}=\frac{1}{\gamma}$, ver equação 2 A na secção 7.2

$$
\text { Portanto: } L=\frac{L_{0}}{\gamma} \quad \text { Como: } \gamma \geq 1 \quad \text { teremos: } \quad L_{\text {movimento }} \leq L_{\text {repouso }}
$$

Logo, quando um corpo está em movimento, ele sofre uma contração na direção do movimento.

Nota-se que para baixas velocidades $v / c$ e $v^{2} / c$ se tornam desprezíveis, tendo em vista que a velocidade $c$ é muito elevada. Em baixas velocidades $\gamma \cong 1$ e as fórmulas da Mecânica clássica são válidas. Porém, para o estudo de partículas em altas velocidades, como elétrons em átomos, ou nos raios cósmicos, devemos usar o fator de Lorentz, também chamado de relativístico.

\subsubsection{Exemplo ilustrativo}

Em outubro de 1977, J. Hafele e R. Keating realizaram uma série de experiências a fim de comprovar dois efeitos relativísticos: a dilatação do tempo e o desvio para o vermelho gravitacional. ${ }^{5} \mathrm{~A}$ ideia se parece com o chamado "paradoxo dos gêmeos" da relatividade especial, no qual dois gêmeos idênticos são separados, o primeiro viajando no espaço sideral e retornando após longo intervalo, para se descobrir

\footnotetext{
Da relatividade geral.
} 
mais jovem que seu gêmeo em casa. Foram utilizados relógios atômicos de césio $C S^{133}$ (ver Seção 2.1.6 em Quartzo, assinalado como o 7), nos quais ocorre dilatação de tempo ou diminuição rítmica do relógio viajante em relação a seu gêmeo em repouso. Foram colocados quatro relógios atômicos portáteis em aviões comerciais que deram duas vezes a volta em torno da Terra, uma vez em cada sentido. Dentro dos erros experimentais de \pm 20 nanoseg, ou seja, bilionésimos de segundos, $10^{-9} \mathrm{~s}$, atribuídos a imprecisões nos dados de voo e a variações intrínsecas dos ritmos dos relógios de césio, as observações concordaram com as previsões.

Por exemplo, para fixar essa ideia, a pessoa que se move junto com o relógio registra o tempo próprio $\Delta t_{0}$, pois o relógio está em repouso em relação a ela. O observador parado, que acompanha o movimento do relógio, registra um tempo dilatado $\Delta t$ para o relógio. Supondo que a leitura do observador parado seja o dobro do tempo próprio, $\Delta t=2 \Delta t_{0}$, e sabendo que $\Delta t=\gamma \Delta t_{0}$, teremos: $\gamma=2=\frac{1}{\sqrt{1-\beta^{2}}} \quad \therefore$ $4\left(1-\beta^{2}\right)=1 \therefore \beta=\sqrt{3 / 4}=0,866$

$$
\text { Como } \beta=v / c \quad v=\beta c=0,866 c
$$

Logo, o relógio deve estar se deslocando com aproximadamente $87 \%$ da velocidade da luz. Essa velocidade equivale a circular pelo equador da Terra 6,5 vezes por segundo.

\subsection{ANÁLISE DOS INTERVALOS DE EVENTOS $d S$}

Como $d x^{2}+d y^{2}+d z^{2}=c^{2} d t^{2}$, então $d s^{2}=c^{2} d t^{2}-d x^{2}-d y^{2}-d z^{2}=0$

(Ver Equação 1 secção 7.2, na Relatividade do tempo)

Como vimos na Seção 5.25.1, Equações 1a e 3, em “Quadripotencial”, obtemos: Ação S :

$$
\begin{aligned}
& \mathrm{S}=-\mathrm{m}_{0} \mathrm{c} \int_{\mathrm{a}}^{\mathrm{b}} \mathrm{ds}=-\mathrm{m}_{0} \mathrm{c}^{2} \int_{\mathrm{t}_{1}}^{\mathrm{t}_{2}} \sqrt{1-\beta^{2}} \mathrm{dt} \\
& \therefore \mathrm{ds}=\mathrm{cdt} \sqrt{1-\beta^{2}}, \log \mathrm{o}: \mathrm{s}=\mathrm{f}(\mathrm{t}) \quad(1) \text {, ver secção } 7.2 \text { equação } 1 \mathrm{~A}
\end{aligned}
$$

$\mathrm{Em} \mathrm{s}_{0}$, ds $=0$

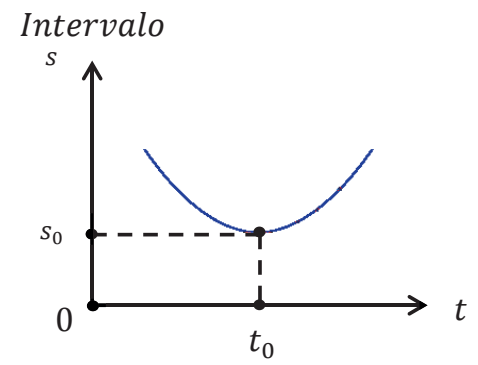


Isto é, $\mathrm{s}_{0}$ é um mínimo, que é um extremo. Pelo princípio da ação mínima, se $s=f(t)$ para que $s$ seja um mínimo em $t=t_{0}$, é necessário que $d s=0$, mas que $d t$ não se anule [ver monografia do autor sobre princípio da ação mínima; Anexo 3, Equação (3) e texto assinalado como 5].

A velocidade da luz $c$ é igual à $\tan \alpha$ que fornece a inclinação da reta $x=c t$ em relação ao eixo dos $t$ :

$$
c=\tan \alpha=\frac{x}{t} \quad \therefore x=c t(1 \mathrm{a}) \quad x^{2}=c^{2} t^{2}
$$

A origem 0 determina a condição presente. A região acima do eixo dos $x$, no sentido positivo do eixo dos t, será o futuro, e a região abaixo, no sentido negativo de $t$, foi o passado.

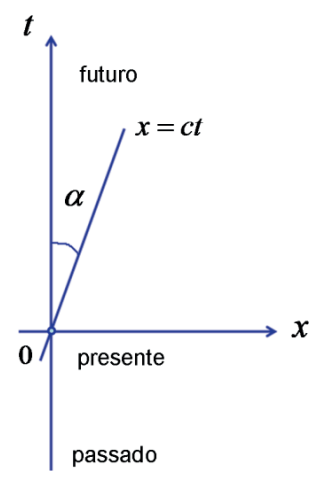

Como a velocidade $c$ é o limite possível de $v$, a região entre o eixo dos $t$ e a reta $x=c t$ é a que pode ser ocupada para movimento dos corpos. A região entre a reta $x=c t$ e o eixo dos $x$ não pode ser ocupada. ${ }^{6} \mathrm{O}$ sinal luminoso percorre a reta $x=c t$. Qualquer outro corpo percorrerá uma linha situada entre essa reta e o eixo dos $t$.

Se considerarmos um espaço bidimensional $x y$, teremos uma superfície de revolução cuja geratriz é a reta $x=c t$, isto é, uma superfície cônica passando pela origem, de equação: $x^{2}+y^{2}=c^{2} t^{2}$. Essa superfície é chamada de "cone de luz".

\footnotetext{
Pois nesse caso teríamos $v>c$, que não é possível pelo postulado de Einstein.
} 


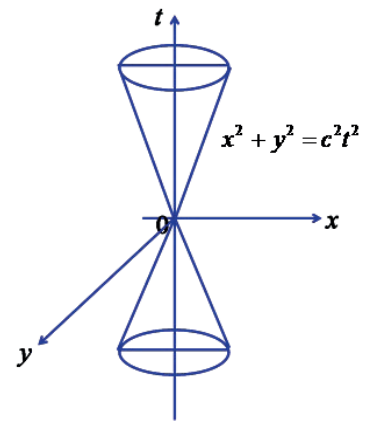

Temos a seguinte situação:

Se $x^{2}+y^{2}-c^{2} t^{2}=0$, o movimento é sobre o cone de luz, e para $x^{2}+y^{2}-c^{2} t^{2} \neq 0$ não está no cone, mas estará dentro dele.

Fora dele, não há a possibilidade de movimento, pois nesse caso $v$ seria maior que $c, v>c$.

Considerando agora o espaço tridimensional $\mathrm{x}, \mathrm{y}, \mathrm{z}$, obteríamos uma hipersuperfície, isto é, não mais uma superfície bidimensional como no caso anterior e sim uma soit disant, uma "superfície" tridimensional em um espaço tetradimensional, conforme Minkowski.

Devemos observar que $t$ é uma coordenada diferente das coordenadas $\mathrm{x}, \mathrm{y}, \mathrm{z}$, pois enquanto nestas podemos nos movimentar para frente e para trás, no eixo dos $t$ só podemos avançar no sentido positivo, pois não se pode recuar no tempo, "avançar" no sentido negativo, a não ser de forma fictícia. Sobre o cone de luz: $x^{2}+y^{2}+z^{2}-c^{2} t^{2}=0$

\subsection{RELATIVIDADE DA MASSA}

Tomando-se o intervalo $d s$, da Seção 7.1, Equação (1):

$$
d x^{2}+d y^{2}+d z^{2}-c^{2} d t^{2}=-d s^{2}
$$

Na Seção 5.26, Equação (5.8b), havíamos antecipado o efeito da velocidade sobre a massa: $m^{\prime}=\frac{m}{\sqrt{1-\beta^{2}}}$

Definindo o vetor "velocidade quadridimensional": $u_{i}=\frac{d x_{i}}{d s}(0)$

E fazendo $x_{4}=c t i$, obtemos: $u_{1}^{2}+u_{2}^{2}+u_{3}^{2}+u_{4}^{2}=-1$ 
Para obter a massa relativística $m^{\prime}$, devemos obter o fator de correção que, multiplicado pela massa de repouso $m_{0}$, conduza a $m^{\prime}$. Para isso, tomamos: $\frac{d u_{i}}{d t}=\frac{d}{d t}\left(\frac{d x_{i}}{d s}\right)=\frac{d}{d t}\left(\frac{d t}{d s} \frac{d x_{i}}{d t}\right)$

Da Seção 7.2, Equação 1a, temos: $\frac{d t}{d s}=\frac{1}{c} \frac{1}{\sqrt{1-\beta^{2}}}=\frac{\gamma}{c}(1)$

Em que $c$ é uma constante, definindo unidades convenientes, podemos fazer que $c$ seja igual a $1{ }^{7}$ então teremos: $\frac{d t}{d s}=\gamma \mathrm{e}$

$\frac{d u_{i}}{d t}=\frac{d}{d t}\left(\gamma \frac{d x_{i}}{d t}\right)$ Para baixas velocidades: $\beta=\frac{v}{c}<<1$ e $\beta^{2} \cong 0$ e $\frac{d u_{i}}{d t}=\frac{d}{d t}\left(\frac{d x_{i}}{d t}\right)$ que concorda com as equações da Mecânica clássica. Logo, a equação da força de Lorentz fica: $\vec{F}=m_{0} \frac{d u_{i}}{d t} \vec{e}_{i}=\frac{d}{d t}\left(m_{0} \gamma \frac{d x_{i}}{d t} \vec{e}_{i}\right)=q(\vec{E}+\vec{v} \times \vec{B})$ como $\vec{v}=\frac{d x_{i}}{d t} \vec{e}_{i}$ e comparando com a equação: $\vec{F}=\frac{d}{d t}\left(m^{\prime} \vec{v}\right)=q(\vec{E}+\vec{v} \times \vec{B})$ teremos: $m^{\prime}=m_{0} \gamma$, explicitamente: $m^{\prime}=\frac{m_{0}}{\sqrt{1-\beta^{2}}}$.

Como já vimos, $\gamma$ é o fator de Lorentz, ver Seção 7.1, Equação (0), e Seção 7.4, Equação (1).

Podemos chegar ao mesmo resultado por mudança de variável. Trocamos a variável tempo $t$ em $\mathrm{s}$ pela variável $\tau=c t$, a distância que a luz viaja em $1 \mathrm{~s}$, chamada de segundo-luz.

Recordemos que $c$ é uma constante. Então podemos escrever: $\frac{d u_{i}}{d \tau}=\frac{d}{d \tau}\left(\frac{d x_{i}}{d s}\right)=\frac{d}{d \tau}\left(\frac{d \tau}{d s} \frac{d x_{i}}{d \tau}\right)$

Como vimos, $\frac{c d t}{d s}=\gamma$, ou seja, $\frac{d \tau}{d s}=\gamma$

Portanto: $\frac{d u_{i}}{d \tau}=\frac{d}{d \tau}\left(\gamma \frac{d x_{i}}{d \tau}\right)$

Multiplicando por $\quad c^{2}: \quad c^{2} \frac{d u_{i}}{d \tau}=c^{2} \frac{d u_{i}}{c d t}=c^{2} \frac{d}{c d t}\left(\gamma \frac{d x_{i}}{c d t}\right) \quad$ Multiplicando por $\quad m_{0}$ : $c^{2} m_{0} \frac{d}{c d t}\left(\gamma \frac{d x_{i}}{c d t} \vec{e}_{i}\right)=\vec{F}$, comparando com a equação da força em função da massa relativística $m^{\prime}$, como vimos:

$\vec{F}=\frac{d}{d t}\left(m^{\prime} \vec{v}\right)$ e tendo em vista: $\vec{v}=\frac{d x_{i}}{d t} \vec{e}_{i}$, conclui-se que: $m^{\prime}=\gamma m_{0}=\frac{m_{0}}{\sqrt{1-\beta^{2}}}$, como $\beta$ é $=v$ sobre c.

Alternativamente, em vez de usar $t$ em seg, poderíamos usar $\tau=c t$ em segundo-luz e obteríamos: $d \tau / d s=\gamma$. 
$\therefore$ A massa varia com a velocidade, ver anexo A 3, secção A 3.1.1, equação 4b.

Pelo princípio da ação mínima, verificamos na Seção 5.25, Quadripotencial, Equação (3a1): Lagrangeana: $\mathrm{L}=-\mathrm{m}_{0} \mathrm{c}^{2} \sqrt{1-\mathrm{v}^{2} / \mathrm{c}^{2}}$

Tendo em vista do Anexo 3, Equação (2a):

Quantidade de movimento: $\mathrm{p}=\frac{\mathrm{dL}}{\mathrm{dv}}$

Derivando L em v: $\frac{d \mathrm{~L}}{\mathrm{dv}}=-\mathrm{m}_{0} \mathrm{c}^{2}\left(1-\mathrm{v}^{2} / \mathrm{c}^{2}\right)^{-1 / 2}\left(-2 \mathrm{v} / \mathrm{c}^{2}\right)=\frac{\mathrm{m}_{0} \mathrm{v}}{\sqrt{1-\beta^{2}}}$

$\mathrm{m}_{0} \mathrm{v}$ é a quantidade de movimento clássica que se obtém quando $\mathrm{v} \ll \mathrm{c}$

Para obter a força, derivamos em t:

$\mathrm{F}=\frac{\mathrm{dp}}{\mathrm{dt}}=\frac{\mathrm{m}_{0}}{\sqrt{1-\beta^{2}}} \frac{\mathrm{dv}}{\mathrm{dt}}$ a expressão $\mathrm{F}=\frac{\mathrm{dp}}{\mathrm{dt}}$, será válida quando a força $\mathrm{F}$ e a velocidade $\mathrm{v}$, forem perpendiculares entre si.

Quando F e v forem colineares, o denominador: $\sqrt{1-\beta^{2}}$ deverá ser elevado a $3 / 2$ em lugar de $1 / 2$ da raiz quadrada (LANDAU).

Resultado obtido anteriormente, porém sem recorrer a nenhum artifício.

Reformulando a equação da força de Lorentz: $\vec{F}=m_{0} \frac{d}{d t}(\gamma \vec{v})=q(\vec{E}+\vec{v} \times \vec{B})$

Daqui tiramos: $\frac{q}{m_{0}}=\frac{1}{\vec{E}+\vec{v} \times \vec{B}}\left[\frac{d}{d t}(\gamma \vec{v})\right]$, relação entre carga e sua respectiva massa. A carga é constante e $m_{0}$ é a massa de repouso, constante.

\subsubsection{Exemplo ilustrativo: a descoberta do elétron}

Em 1897, Joseph John Thomson (1856-1940), aluno de Maxwell, determinou o quociente $\mathrm{e} / \mathrm{m}$ do elétron. Para isso utilizou um tubo de raios catódicos, "avô" dos atuais tubos de televisão.

O tubo de raios catódicos é uma ampola de vidro onde se faz vácuo da ordem de $10^{-4}$ ou $10^{-5} \mathrm{mmHg}$. 


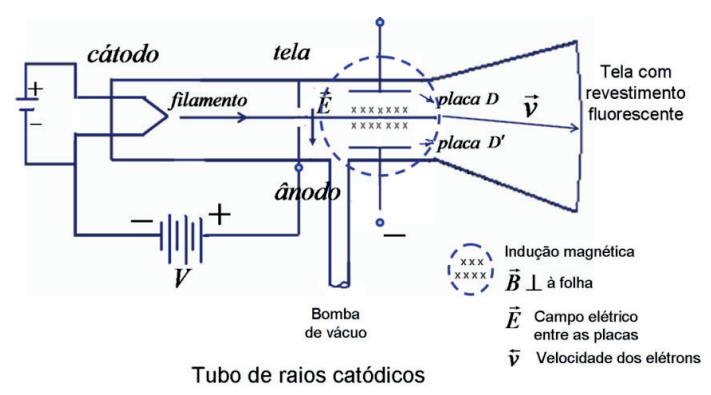

O filamento forma o catodo e a tela, o ânodo. Entre os dois existe uma diferença de potencial da ordem de alguns milhares de volts .

Um estreito feixe se forma e incide na tela fluorescente, indicando um ponto de incidência. Esse feixe era denominado "raio catódico". Thomson, que realizou as experiências no Cavendish Laboratory em Cambridge, mostrou que os raios são feixes de partículas com cargas negativas, que chamou de “corpúsculos”. George Johnstone Stoney (1826-1911), afirmou, em 1874, que a eletricidade era devida a esses corpúsculos, aos quais, em 1891, deu o nome de elétrons. Thomson considerou que faziam parte da matéria.

Na Seção 5.5, Equação 1, vimos que a existência de um campo elétrico $\vec{E}$ entre as placas $D$ e $D^{\prime}$ faz a carga do elétron $e$ ser sujeita a uma força $\vec{F}_{1}=e \vec{E}$. Isso faz o feixe defletir para cima. Se aplicarmos uma indução magnética $\vec{B}$, perpendicular a $\vec{E}$, isto é, fazendo com que os polos $N$ e $S$ fiquem alinhados em uma direção perpendicular ao eixo do tubo, por exemplo, com um imã em ferradura ou um solenoide equivalente.

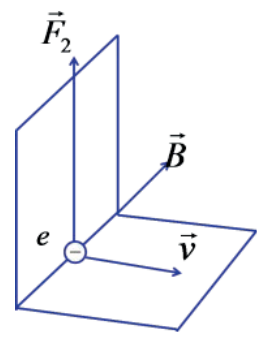

De acordo com a Seção 5.11, Equação (1), utilizando-se uma indução $\vec{B}$ que provoque no elétron uma força $\vec{F}_{2}{ }^{8}$ com módulo igual ao de $\vec{F}_{1}$, mas de sentido oposto, compensando a deflexão ocasionada

${ }^{8}$ Da introdução para o estudo da teoria da relatividade 1 do autor: $\vec{F}_{2}=e v \vec{B}$, em que $v$ é a velocidade dos elétrons no feixe eletrônico. 
por $\vec{F}_{1}$, o feixe eletrônico volta à posição inicial. Os vetores $\vec{E}, \vec{B}$ e $\vec{v}$ são mutuamente perpendiculares entre si. Teremos $\left|\vec{F}_{1}\right|=\left|\vec{F}_{2}\right|$ e $e E=e v B$, ou seja, $v=E / B$. Assim, podemos determinar $\vec{v}$.

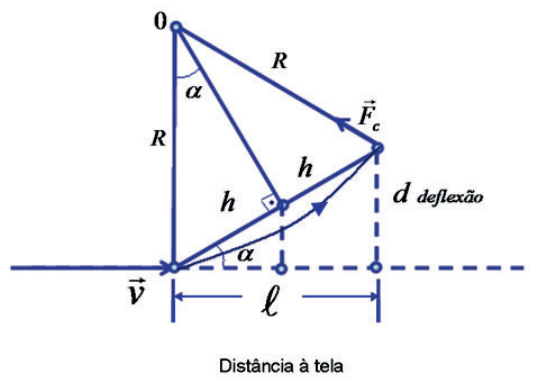

Anula-se o campo elétrico. O feixe de elétrons descreverá um arco de círculo no campo magnético. Os elétrons ficarão sujeitos à força centrípeta, isto é, dirigida para o centro: $\vec{F}_{c}$. Pela $2^{\text {a }}$ lei de Newton temos, em módulo: $F_{c}=m a_{r}$ em que $a_{r}$ é a aceleração radial, cujo valor, como já sabemos é: $a_{r}=\frac{v^{2}}{R}$. Portanto: $\vec{F}_{c}=\frac{m v^{2}}{R}$

Essa força é devido à ação da indução magnética sobre o elétron, como já vimos: $F_{c}=e v B$

Logo: $e v B=\frac{m v^{2}}{R} \quad \therefore \frac{e}{m}=\frac{v}{R B}$

Combinando com: $v=\frac{E}{B}$, temos $\frac{e}{m}=\frac{E}{R B^{2}}$

Da trigonometria, podemos dizer: $\operatorname{sen} \alpha=\frac{d / 2}{h}=\frac{h}{R} \quad \therefore R=\frac{h^{2}}{d / 2}$

Pelo teorema de Pitágoras: $h^{2}=(\ell / 2)^{2}+(d / 2)^{2}$

Combinando as duas expressões: $R=\frac{\ell^{2}+d^{2}}{4}\left(\frac{2}{d}\right)=\frac{\ell^{2}+d^{2}}{2 d}$

Assim, obteremos: $\frac{e}{m}=\frac{2 d}{\ell^{2}+d^{2}}\left(\frac{E}{B^{2}}\right)$ 
A velocidade $v$ do elétron $^{9}$ é da ordem de $1 / 10 c$. Então, o valor de $\gamma$ será: $\gamma=\frac{1}{\sqrt{1-\beta^{2}}}, \beta=\frac{v}{c}=0,1$ $\therefore \gamma=\frac{1}{\sqrt{1-0,01}}=1,005$, isto é, a massa $m$ será $0,5 \%$ maior que a massa de repouso $m_{0}$. Esse efeito relativístico deve ser levado em conta na fórmula anterior.

\subsection{COMPROVAÇÃO DA RELATIVIDADE DA MASSA}

Bucherer, em 1909, testou a relatividade de massa, medindo $e / m$ em função de $v$.

Uma fonte $S$ contendo radio $R a^{228}$, emite raios $\beta$, que são elétrons rápidos, com velocidade $v$ com uma pequena porcentagem menor do que a da luz. Atravessam um condensador longo comparado com a distância entre as placas e atingem a placa fotográfica $P$. O aparelho é evacuado e está contido em um forte campo magnético cujo vetor indução é perpendicular à direção do movimento dos elétrons e ao vetor campo elétrico entre as placas. O princípio de funcionamento é o mesmo do equipamento precedente, contudo, os raios catódicos são substituídos por raios $\beta$, e a tela fosforescente, por uma placa fotográfica.

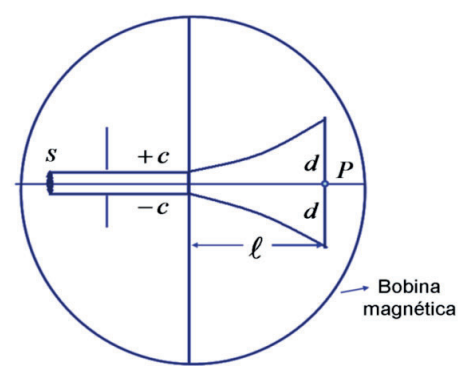

A intensidade da indução magnética é ajustada de modo que, para uma determinada velocidade $v$ a deflexão magnética seja compensada pela deflexão elétrica. Nessas condições, temos: $v=\frac{E}{B}$ como no caso anterior.

Quando os elétrons estiverem livres do condensador, ficam sujeitos somente ao campo magnético e sua trajetória será um arco de círculo no qual a indução magnética provoca uma força centrípeta. Como vimos, obtemos: $\frac{e}{m}=\frac{v}{R B}$, inserindo $v=\frac{E}{B}$ teremos: $\frac{e}{m}=\frac{E}{R B^{2}}$.

\footnotetext{
Nos raios catódicos.
} 
Se invertermos os campos elétrico e magnético, o feixe de elétrons defletirá na direção oposta e com o mesmo valor $d$. Como anteriormente, podemos calcular $R$ por considerações geométricas: $R=\frac{\ell^{2}+d^{2}}{2 d}$, resultando: $\frac{e}{m}=\frac{2 d}{\ell^{2}+d^{2}}\left(\frac{E}{B^{2}}\right)$.

Bucherer encontrou que, conforme a velocidade o valor de $e / m$ variava conforme: $e / m=\frac{e}{\gamma m_{0}}$, e como a carga é invariável: $m=\gamma m_{0} \quad \therefore e / m$ só varia com a massa.

$$
\gamma \text { é o fator de Lorentz: } \gamma=\frac{1}{\sqrt{1-\beta^{2}}}, \beta=\mathrm{v} / \mathrm{c} \text {, ver Seção 7.2, Equação }(0) \text {. }
$$

Das experiências de eletrolise de Faraday em 1833, verifica-se a quantidade de eletricidade para liberar um átomo-grama, ou seja, $1,008 g$ de $H$, é denominada Faraday, abreviadamente $F$.

Essa quantidade é igual a: $F=96.487 C$

Como resultado das experiências de Thomson (BORN), temos: $\frac{e}{m_{0}}=1840 F$ (1)

Um átomo-grama contém $N$ átomos. E $N$ é o número de Avogadro.

A massa de um átomo de $H$ será: $m_{H}=\frac{1,008}{N}$

A carga elementar do elétron " $e$ " multiplicada por $N$ fornecerá a carga necessária para liberar 1 átomo-grama de $H$, pois cada elétron provém da separação do elétron do átomo, $\log o F=e N$

$$
\begin{gathered}
F=e N=1,008 \frac{e}{m_{H}}, \quad \text { comparando com: } \frac{e}{m_{0}}=1840 F, \text { obtemos: } m_{0}=\frac{m_{H}}{1,008 \times 1840} \\
\frac{\mathrm{e}}{\mathrm{m}_{0}}=1840 \times 1,008 \frac{\mathrm{e}}{\mathrm{m}_{\mathrm{H}}} \Rightarrow \mathrm{m}_{0}=\frac{\mathrm{m}_{\mathrm{H}}}{1,008 \times 1840}
\end{gathered}
$$

Então, tiramos a relação entre $m_{0}$ e $m_{H}$.

Da eletrólise de uma solução de $\mathrm{AgNO}_{3}$ resulta a primeira definição do Ampère, o Ampère internacional. Seja uma solução de $\mathrm{AgNO}_{3}$ com eletrodos de $\mathrm{Ag}$, teremos: $\mathrm{AgNO}_{3} \rightarrow \mathrm{Ag}^{+}+\mathrm{NO}_{3}^{-}{ }^{10}$

${ }^{10}$ Para mais detalhes, ver Anexo 11, sob título Eletrólise, secção A 11.2.2. 


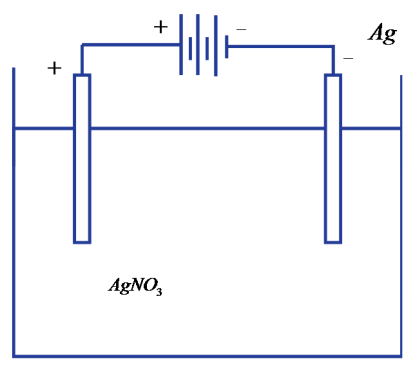

Conectando os eletrodos a uma bateria, os íons $\mathrm{Ag}^{+}$se dirigirão ao eletrodo negativo onde se neutralizarão com a carga de elétrons e se depositarão.

Para depositar 1 átomo-grama de $A g$, ou seja; 107,88g de $A g$, será necessário passar uma carga de $1 F=96.487 C$, logo a quantidade de $A g$ depositada para uma carga de $1 C$ será: $\frac{107,88}{96.487}=0,001118 \mathrm{~g} / \mathrm{C}$ ou $1,118 \mathrm{mg} / \mathrm{C}=$ Coulomb, unidade de carga elétrica, ver Seção 5.1 página inicial.

Ampère internacional é a corrente constante que faz depositar $1,118 \mathrm{mg}$ de $\mathrm{Ag}$ por segundo de uma solução de $\mathrm{AgNO}_{3} .1 \mathrm{~A}$ internacional=0,99986 A absoluto, definido como se viu em função da força magnética que surge entre dois condutores de corrente elétrica. ${ }^{11}$ É medida com a balança de corrente eletrodinâmica.

\subsection{MEDIÇÃO DA CARGA ELEMENTAR $e$}

O método usado por Townsend (1897) e Thomson (1896) foi aperfeiçoado por Charles Thomson Rees Wilson em 1903. Uma câmara cilíndrica, dotada de um pistão, sofre uma expansão de forma que o vapor d'água presente fique supersaturado. Os íons presentes agem como núcleos de condensação onde o vapor d'água condensa, formando gotículas. É a chamada "Câmara de detecção ou de bolhas de Wilson" (LAROUSSE). Dentro da câmara, colocam-se duas placas paralelas conectadas a uma bateria. Os íons situados entre as placas ficam sujeitos a um campo elétrico. As névoas que se formam sofrem influência da gravidade e do campo elétrico, e o topo da névoa é acompanhado, isto é, a velocidade é medida. Considerando as gotículas só sob efeito da gravidade e aplicando a lei de Stokes da queda de corpos em um meio viscoso, obtêm-se o tamanho e a massa das gotículas. Supondo-se que as gotículas mais carregadas caem mais rapidamente, medem-se somente as do topo. Wilson obteve $e=1,033 \times 10^{-19} \mathrm{C}$, incerteza no resultado.

Robert Andrews Millikan (1869-1953), entre 1909 e 1917, aperfeiçoou o método de Wilson, pulverizando gotículas de óleo em vez gotículas de água, evitando os erros ocasionados pela evaporação desta última.

\footnotetext{
${ }^{11}$ Ver Seção 5.24 .
} 
Quando um corpo esférico cai livremente em um meio viscoso, ele acelera até uma velocidade terminal $v_{g}$, quando a força agindo no corpo se iguala à força viscosa. Sendo $m$ a massa aparente: $m g=k v_{g}$ sendo $k$ uma constante de proporcionalidade.

Sendo $E$ o campo elétrico agindo contra a gravidade, resultando uma velocidade terminal $v_{E}$ : $q E-m g=k v_{E}$.

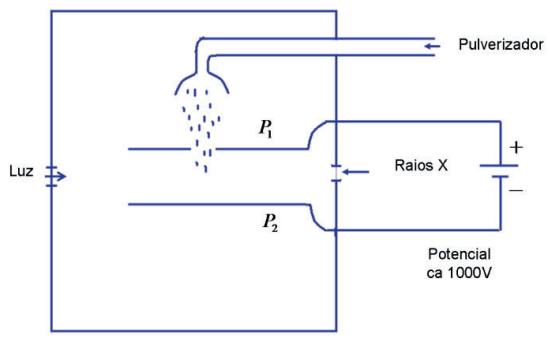

Relação entre as velocidades: $\frac{v_{g}}{v_{E}}=\frac{m g}{q E-m g}$

A carga $q$ será: $q=\frac{m g}{E} \frac{\left(v_{g}+v_{E}\right)}{v_{g}}$

Se a gota captar outro íon, por radiação cósmica ou raios $X$, resultará em outra carga $q^{\prime}$.

A variação $\Delta q$ será: $\Delta q=q^{\prime}-q=\frac{m g}{E} \frac{\left(v_{E}^{\prime}-v_{E}\right)}{v_{g}}$.

Millikan observou que existia um mínimo divisor comum entre as cargas para uma mesma gota e esse menor valor da carga ele identificou com o valor do elétron de Stoney. Isso significa uma quantização da carga elétrica. ${ }^{12}$

Para achar $m$, aplicamos a lei de Stokes: $4 / 3 \pi a^{3} g\left(\rho-\rho^{\prime}\right)=6 \pi \eta a v_{g}$ em que $a$ é o raio da esfera, $\rho$ a densidade da gota, $\rho^{\prime}$ a densidade do meio e $\eta$ é a viscosidade do meio.

A partir daqui, se tem que: $a=\left(\frac{9}{2} \frac{\eta v_{g}}{g\left(\rho-\rho^{\prime}\right)}\right)^{1 / 2}$

\footnotetext{
${ }^{12}$ Portanto: $q=n e$, em que $n$ é um número inteiro, nunca fracionário.
} 
Quando as gotas são muito pequenas, $v_{g}$ deve ser multiplicado por um fator de correção: $v_{g}^{\prime}=v_{g}\left(1+\frac{b}{p a}\right)$, em que $p$ é a pressão do ar, e $a$ e $b$ são constantes determinadas experimentalmente.

Millikan obteve: $e=1,5913 \times 10^{-19} C$, o que resultou em desacordo com outras medições indiretas. A discrepância era causada pela viscosidade usada por Millikan. Após a correção, o resultado foi: $e=1,6012 \times 10^{-19} \mathrm{C}$, concordando com valores obtidos por outros métodos.

Daqui resulta: $N=6,02472 \times 10^{23}$ átomos/átg (número de Avogadro), como vimos na Seção 7.5, Equação (1), Thomson obteve $\mathrm{e} / \mathrm{m}_{0}=1840 \mathrm{~F}$, da eletrólise da solução de $\mathrm{AgNO}_{3}, 1 \mathrm{~F}=96487 \mathrm{C}, \mathrm{m}_{\mathrm{H}}=$ $1,008 / \mathrm{N} \mathrm{e}^{\mathrm{m}_{\mathrm{H}}} / \mathrm{m}_{0}=1,008 \times 1840$, relação entre as massas do $\mathrm{H}$ e do elétron. ${ }^{13}$

\subsection{RELAÇÃO ENTRE MASSA E ENERGIA}

A variação da massa com a velocidade conduz à modificação de nossas ideias sobre energia. Definindo a força como variação da quantidade de movimento:

$F=\frac{d}{d t}(m v)=m \frac{d v}{d t}+v \frac{d m}{d t}$, e a energia cinética como o resultado da ação da força provocando um deslocamento: $d E=F d x$

Inserindo a força: $d E=m \frac{d v}{d t} d x+v \frac{d m}{d t} d x=m v d v+v^{2} d m$

Como: $m=\frac{m_{0}}{\left(1-v^{2} / c^{2}\right)^{1 / 2}}$, diferenciando: $d m=\frac{m_{0}}{c^{2}} \frac{v d v}{\left(1-v^{2} / c^{2}\right)^{3 / 2}}=\frac{m v d v}{c^{2}-v^{2}} \quad$, pois:

$\frac{\mathrm{dm}}{\mathrm{dv}}=\mathrm{m}_{0}\left(-\frac{1}{2}\right)\left(1-\mathrm{v}^{2} / \mathrm{c}^{2}\right)^{(-1 / 2-1)}\left(-2 \mathrm{v} / \mathrm{c}^{2}\right)=\frac{\mathrm{m}_{0}}{\mathrm{c}^{2}\left(1-\mathrm{v}^{2} / \mathrm{c}^{2}\right)^{1 / 2}} \frac{\mathrm{v}}{\left(1-\mathrm{v}^{2} / \mathrm{c}^{2}\right)}$, porém:

$\mathrm{m}=\frac{\mathrm{m}_{0}}{\left(1-\mathrm{v}^{2} / \mathrm{c}^{2}\right)^{1 / 2}}$ e $1-\mathrm{v}^{2} / \mathrm{c}^{2}=\frac{\mathrm{c}^{2}-\mathrm{v}^{2}}{\mathrm{c}^{2}}$

Substituindo $m v d v$ em $d E$, resulta: $d E=c^{2} d m$ (a), integrando: $\int_{0}^{E_{k}} d E=c^{2} \int_{m_{0}}^{m} d m$, obtemos: $E_{k}-E_{0}=c^{2}\left(m-m_{0}\right), E_{0}$ é energia quando $v=0$, é nula: $E_{0}=0, m_{0}$ é massa de repouso para $v=0,{ }^{14}$ $\operatorname{logo} E_{k}=c^{2}\left(m-m_{0}\right)(0)$

Chamemos a variação de massa de $\Delta m=m-m_{0}$, então: $E_{k}=c^{2} \Delta m$.

\footnotetext{
${ }_{13}$ Por meio das experiências de Faraday, deduziu: $F=a^{Q} / M$, em que $a=A / z$, sendo $A$, massa atômica e $z$, valência, relacionase as massas de $A g$ e $H$, com a carga $Q$ passando pela solução. Para mais detalhes, ver Anexo 11, "Eletrólise”, secção A11.4.

${ }^{14}$ Quando a velocidade for nula, ou seja, corpo em repouso.
} 
Isto é, quando o corpo está em movimento com velocidade $v$, sua massa é acrescida de um valor $\Delta m=\frac{E_{k}}{c^{2}}$, sendo $E_{k}$ energia cinética do corpo. A equação $E_{k}=c^{2} \Delta m$ foi estabelecida por Einstein em 1905.

Na secção 7.15 daremos uma demonstração baseada na pressão da radiação.

As leis da conservação da massa e a da conservação da energia tornam-se uma só.

\subsubsection{Exemplos ilustrativos}

\subsubsection{Fissão nuclear}

Em 1939, Lise Meitner e Otto Frisch mostraram que um núcleo $U^{235}$, quando bombardeado com nêutron térmico, pode se dividir, com emissão de energia, em duas partes aproximadamente iguais. Frisch denominou o processo de fissão.

Uma reação típica seria: $U^{235}+n^{1} \rightarrow C e^{140}+Z r^{94}+2 n^{1}$

Os fragmentos da fissão podem ter seus números de massa variando de 72 a 158 (KAPLAN). Como na reação há geração de dois nêutrons, existe a possibilidade destes bombardearem outros núcleos de $U^{235}$, e a reação se tornar em cadeia.

Fazendo um balanço das massas envolvidas na reação, verificamos que a massa final é menor que a massa de $U^{235}$ :

$$
\Delta m=(235,0439 u)-(139,9054 u+93,9063+1,00867 u)=0,224 u
$$

Sendo $u$ a unidade de massa atômica.

Essa diferença de massa é convertida em energia cinética de acordo com a fórmula: $E=c^{2} \Delta m$. Assim, temos: $E(\operatorname{ergs})=m(g) \times\left(2,998 \times 10^{10}\right)^{2} \mathrm{~cm}^{2} / \mathrm{s}^{2}=m(g) \times 8,99 \times 10^{20} \mathrm{~cm}^{2} / \mathrm{s}^{2}$

$$
1 M e v=1,602 \times 10^{-6} \text { erg } \quad E(\mathrm{Mev})=m(g) \times \frac{8,99 \times 10^{20}}{1,602 \times 10^{-6}}=m(g) 5,61 \times 10^{26}
$$

$1 u=1,66 \times 10^{-24} \mathrm{~g}$

Portanto: $E(M e v)=m(u) \times 1,66 \times 10^{-24} \times 5,61 \times 10^{26}=m(u) \times 932$

Logo, a energia resultante será: $E=c^{2} \Delta m=(0,224 u) \times(932 \mathrm{Mev} / u)=209 \mathrm{Mev}$

A reação em cadeia do $U^{235}$ para se autossustentar necessita de um valor mínimo de massa, isto é, a massa crítica. 
Nos reatores térmicos, o núcleo do reator contém o moderador, onde se intercalam os elementos combustíveis. O moderador freia os nêutrons para obter uma reação controlada que, de outra forma, seria explosiva como uma bomba atômica. O moderador pode ser água pesada, carbono em forma de grafite ou água leve. O combustível deve ser protegido com um envoltório conveniente, pois o urânio e o plutônio são muito ativos quimicamente (MURRAY, BABCOCK, WILCOX) e são atacados pelo ar, água e metais líquidos, os refrigerantes comumente usados. Também previne o escape dos produtos de fissão e outros materiais tóxicos do elemento combustível para o fluido refrigerante. O fluido refrigerante retira o calor do reator, para evitar o superaquecimento e para aproveitar o calor gerando energia, por intermédio de um trocador de calor que gera vapor para movimentar uma turbina, gerando energia mecânica. O fluido refrigerante pode ser $\mathrm{CO}_{2}$, sob pressão, água pesada sob pressão, água leve ou sódio líquido.

Há dois itens de primordial importância para a segurança do reator: o sistema de controle e a blindagem. O primeiro consiste de barras ou tiras de material absorvente de nêutrons, montados no núcleo de forma que possam ser movidos para dentro e para fora de modo rápido e preciso. Os materiais geralmente empregados são os que têm uma elevada secção de choque de absorção de nêutrons, como boro, háfnio e cádmio. As blindagens devem cercar inteiramente o reator para absorver nêutrons penetrantes e raios $\gamma$, protegendo, assim, o pessoal de operação. Os nêutrons são mais bem atenuados por água ou outro material hidrogenado como parafina ou polietileno. Os raios $\gamma$ são atenuados por materiais muito densos. O concreto comum é barato e preenche os requisitos, sendo largamente usado juntamente com água ou com chapas de aço, chumbo ou outro metal. São essas blindagens que tornam um reator tão grande.

Em 1979, um acidente afetou seriamente o $2^{\circ}$ reator da usina de Three Mile Island, nos Estados Unidos, porém, sem causar vítimas ou grandes danos ao meio ambiente.

Contudo, em 1986, em Chernobil, na Ucrânia ocorreu a explosão do reator $n^{\circ} 4$. Uma nuvem radioativa cobriu todo o centro-sul da Europa nas semanas seguintes causando danos enormes à economia, principalmente à agricultura. Segundo as autoridades soviéticas, 31 pessoas morreram, mais de 200 ficaram feridas ou foram contaminadas pela radiação e 135 mil habitantes tiveram que ser evacuados. As investigações revelaram: os operadores, embora tivessem experiência em usina convencional, não tinham em uma usina nuclear. Houve falhas no projeto do sistema de controle, cujas hastes eram ocas na extremidade, provocando bolhas de vapor no fluido refrigerante (água), ocasionando que, quando se baixavam as hastes, esperando baixar a potência, ao contrário, esta aumentava; houve então um superaquecimento, as hastes travaram e não foi possível fazer o desligamento do reator, provocando a explosão do vapor. Outro ponto fraco do reator era que a blindagem de sua cobertura estava incompleta. Em consequência, o vapor que tornaria a atmosfera saturada dentro do reator, escapou. Esse vapor teria dissolvido o iodo e o césio radioativos liberados e que foram espalhados na atmosfera. Também o grafite do moderador, devido à entrada de oxigênio no reator, entrou em combustão, queimando por 10 dias. O único jeito foi construir um "sarcófago" de concreto para absorver a radiação e conter o combustível remanescente. O governo soviético embargou a construção dos reatores 5 e $6 .^{15}$

${ }^{15}$ Chernobyl em 04/10/2016. Trinta anos após o incidente, a área em torno, próximo da fronteira entre Ucrânia e Bielorrússia, continua abandonada, com índices elevados de radiação $\gamma$, produzindo graves mutações na vida selvagem, flora e fauna. 
Trata-se de uma "alquimia", no sentido de transformar um elemento em outro. Enquanto os alquimistas estavam mais interessados em transformar chumbo em ouro, estamos nos referindo à transformação do $U^{235}$ em outros elementos diferentes.

A respeito do acidente nuclear em Fukushima, sabe-se que o Japão não tem reservas de combustíveis fósseis nem recursos hídricos para a geração de energia. Por esses motivos optou, desde 1954, para a geração de energia nuclear, limitando apenas para propósitos pacíficos, e seu primeiro reator foi construído pela General Electric do Reino Unido. Na década de 1970, os primeiros reatores a água leve foram construídos em cooperação com as empresas americanas General Electric e Westinghouse Electric Co., com trabalho contratual por companhias japonesas, que mais tarde obteriam licenças para construir usinas similares, e pesquisas foram desenvolvidas desde então. No entanto, sempre houve grande controvérsia quanto à segurança das usinas nucleares, pois o Japão é muito sujeito a atividades sísmicas. Alguns acidentes ocorreram, mas sem grande gravidade, porém, em 11 de março de 2011 ocorreu um terremoto seguido de tsunami, com ondas que chegaram a $23 \mathrm{~m}$. As emissões radioativas obrigaram o desalojamento de 80 mil moradores e afetaram gravemente a agricultura, pecuária e pesca local.

Nos meses seguintes até julho de 2011, dos 54 reatores existentes, 19 estavam em operação, desde quando ocorreu o desastre de Fukushima, aumentando "o risco de uma forte escassez de energia em 2012”. Em 27 de abril, aproximadamente 55\% do combustível no reator da unidade 1 de Fukushima havia derretido, $35 \%$ do combustível na unidade 2 e $30 \%$ na unidade 3 enquanto os combustíveis superaquecidos usados nas piscinas de armazenamento das unidades 3 e 4, provavelmente também foram danificadas. O acidente ultrapassou em nível de gravidade o de Three Mile Island, de 1979, e é comparável ao de Chernobil, em 1986.

As estrelas, incluindo o Sol, utilizam de maneira natural o processo que chamamos de fusão termonuclear, transformando $H$ em $H e$, também considerada uma espécie de alquimia. A reação pode ser sintetizada por (KAPLAN; HALLIDAY, RESNICK, WALKER): ${ }^{16}$

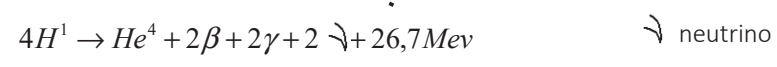

A probabilidade de ocorrer é baixa, mas, como há muito material, acontece por um longo tempo, não sendo, pois, explosiva. No entanto, para a reação ocorrer, é necessária uma temperatura de cerca $10^{8} \mathrm{~K}$ em lugar dos $600 \mathrm{~K}$ que os alquimistas usavam.

Se somarmos a massa de $4 H^{1}$, teremos: 4,03258u e a massa atômica de:

$$
1 \mathrm{He}^{4} \quad 4,00387 \mathrm{u}
$$

Diferença de massa: $\quad 0,02871 \mathrm{u}$, sendo u, unidade de massa atômica.

Usando a equação $E=c^{2} \Delta m$, como fizemos anteriormente, obtemos: $26,7 \mathrm{Mev}$

Essa energia em parte é liberada na forma de irradiação, e outra parte é utilizada para manter a temperatura elevada, dando continuidade à reação.

${ }^{16} 26,7 \mathrm{Mev}$ equivale a $176.119^{\mathrm{Mwh}} / \mathrm{kgdeH}$, fatores de conversão: $1 \mathrm{u}=1,660 \times 10^{-27} \mathrm{~kg}, 1 \mathrm{Mev}=4,450 \times 10^{-23} \mathrm{Mwh}$ 
A bomba de hidrogênio funciona, inicialmente, por meio da explosão de uma bomba de fissão que fornece a temperatura de ignição para o material de fusão, deutério de lítio (ISAACS, PITT), que envolve a bomba de fissão. A reação será: $\quad L i^{6}+D^{2} \rightarrow 2 H e^{4}+22,5 M e v$

A temperatura de ignição é cerca de $45.000 .000^{\circ} \mathrm{K}$.

A fusão controlada ainda se encontra em um estado inicial, apesar dos vários recursos e esforços despendidos até hoje. As dificuldades são grandes, a começar pela elevada temperatura necessária no processo, e considerando que não existe nenhum material que possa suportar essa temperatura. Imaginouse então usar um plasma, a exemplo do existente nas estrelas, contendo deutério e trítio: $D^{2}+T^{3} \rightarrow \mathrm{He}^{4}+n^{1}+17,6 \mathrm{Mev}$

Para evitar o contato material do plasma com as paredes e conseguir uma alta densidade e temperatura, utiliza-se o pinch effect, descoberto por Bennett, que é a constrição ocasionada no plasma pelo campo magnético criado por corrente elétrica. No entanto, está sujeito a instabilidades que desfazem a contração. Uma dessas experiências é o TOKAMAK, acrônimo russo para "câmara magnética toroidal", feita em um toroide circular. Até hoje, não se conseguiu manter a reação, a não ser por poucos $\mu s$.

Outra técnica é a do "confinamento inercial", em que pequenas pelotas de $D T$ são comprimidas pelo ataque multilateral de feixes de laser ou partículas. A temperatura se eleva até $10^{8} \mathrm{~K}$ e a densidade de partículas aumenta por um fator de cerca de $10^{3}$, podendo causar a fusão termonuclear (HALLIDAY, RESNICK, WALKER).

\subsection{CONTRIBUIÇÃO DE HENRI POINCARÉ (1854-1912)}

Apesar de pouco lembrado, não podemos deixar de mencioná-lo. Sua maior contribuição na FísicaMatemática foi a publicação sobre dinâmica do elétron, publicada em 1906, na qual obteve muitos resultados da teoria especial da relatividade independentemente de Einstein. A principal diferença foi que Einstein desenvolveu a teoria de considerações elementares relativas a sinais luminosos, enquanto Poincaré se baseou na teoria completa do eletromagnetismo, restrita aos fenômenos associados com esta última (ENC. BRITANNICA).

\subsubsection{Exemplos ilustrativos}

Da $2^{\mathrm{a}}$ lei de Newton temos: $F=\frac{d p}{d t}$, onde $p=m v$, é a quantidade de movimento.

$$
\text { Logo: } \int_{p_{0}}^{p} d p=\int_{t_{0}}^{t} F d t \quad \therefore p-p_{0}=\int_{t_{0}}^{t} F d t=I
$$

I é chamada Impulsão.

A variação da quantidade de movimento é igual à impulsão.

Pela $3^{\text {a }}$ lei de Newton: a toda ação corresponde uma reação igual e contrária, então: $F=-F^{\prime}$. A propulsão a jato baseia-se nessa lei. 
No caso de motores a jato: $\mathrm{m}_{2} \mathrm{v}_{2}-\mathrm{m}_{1} \mathrm{v}_{1}=\mathrm{F} \Delta \mathrm{t}=\mathrm{I}$, isto é, a impulsão é igual à força de empuxo multiplicada pelo intervalo de tempo. Considerando o volume de controle $V C$ do ar entrando no compressor do motor, com massa $m_{1}$ e velocidade $v_{1}$, passando pelas câmaras de combustão, onde parte do ar queima o combustível querosene ou gasolina de aviação, os gases de combustão resultantes mais o excesso de ar se expandem com a elevação de temperatura e saem pelo bocal de escapamento com massa $m_{2}$ e velocidade $v_{2}>v_{1}$, havendo, portanto, uma aceleração da massa gasosa. Da entrada até a saída transcorre um intervalo de tempo $\Delta t$. A força $F$, resultante dessa variação da quantidade de movimento no intervalo $\Delta t$, para trás, origina uma reação no motor igual a “ $-F$ ”, que propulsiona o avião para frente.

No caso de um foguete, consideramos o $V C$ como a massa de gás formada na câmara do foguete por um monopropelente ou bipropelente, cuja massa será $m_{1}$ e velocidade $v_{1}$, que é a do foguete com relação à Terra. No bocal de escapamento, teremos a massa $m_{2}=m_{1}$, mas a velocidade será $v_{2}$, acelerada pela expansão devido à alta temperatura e pelo formato do bocal. O propelente pode ser sólido ou líquido. Por exemplo, no caso de um monopropelente sólido, pode-se ter pólvora, usada nos foguetes juninos. A reação seria:

$$
2 \mathrm{KNO}_{3}+\mathrm{S}+3 \mathrm{C} \rightarrow \mathrm{K}_{2} \mathrm{~S}+3 \mathrm{CO}_{2}+\mathrm{N}_{2}+550 \mathrm{cal} / \mathrm{g} \text { de reagentes }
$$

O nitrato de $K$ (Salitre do Egito ou da Índia) é o oxidante.

Um monopropelente líquido seria o peróxido de $\mathrm{H}$ ( $\mathrm{H}_{2} \mathrm{O}_{2}$, água oxigenada). Um bipropelente líquido seria a hidrazina + oxigênio líquido.

Os Estados Unidos planejam um foguete nuclear. Um reator nuclear com moderador de grafite, refrigerado a $H$. O $H$ é aquecido no reator a $2.000{ }^{\circ} \mathrm{C}$ e usado como propulsor. Para suportar essa temperatura, o bocal é resfriado pelo $H$ líquido. É nessa forma que se armazena o $H$ no foguete (ISAACS, PITT).

Da relatividade restrita: $p=m v=\frac{m_{0} v}{\sqrt{1-v^{2} / c^{2}}}$

Vimos que a Energia cinética (ver Equação 1, Seção 7.7):

$$
E_{K}=\frac{m_{0} c^{2}}{\sqrt{1-v^{2} / c^{2}}}-m_{0} c^{2}=\left(m-m_{0}\right) c^{2}=c^{2} \Delta m
$$

$m_{0}$ é a massa de repouso, $m_{0} c^{2}$ energia de repouso

A energia total será: $E=E_{K}+m_{0} c^{2}=m c^{2} \quad \therefore m=\frac{E}{c^{2}}$

Combinando com a equação (1): $v=\frac{c^{2} p}{E}$ 


$$
\begin{aligned}
& \operatorname{De}(2): E=\frac{m_{0} c^{2}}{\sqrt{1-v^{2} / c^{2}}}=\frac{m_{0} c^{2}}{\sqrt{1-c^{4} p^{2} / E^{2} c^{2}}}=\frac{m_{0} c^{2}}{\sqrt{1-p^{2} c^{2} / E^{2}}}=\frac{E m_{0} c^{2}}{\sqrt{E^{2}-p^{2} c^{2}}} \\
& \sqrt{E^{2}-p^{2} c^{2}}=m_{0} c^{2} \\
& E=c \sqrt{m_{0}^{2} c^{2}+p^{2}}
\end{aligned}
$$

Um caso particular e interessante ocorre quando a massa de repouso for nula, como em fótons e neutrinos. Teremos (LANDAU): $E=c p$ ou $p=E / c \cdot(2 \mathrm{~b})^{17}$

Cogitou-se a utilização de um foguete fotônico. O espaço cósmico não tendo atmosfera, não ocasionaria resistência à propulsão e a velocidade poderia ser bastante incrementada, considerando que a velocidade dos fótons é a da luz. No entanto, a realização desse projeto ainda apresenta muitas dificuldades.

Mais factível é o foguete de propulsão iônica. Emprega-se um reator nuclear acoplado a um turbogerador para a produção de energia elétrica, empregada para ionizar vapores de césio $C s^{133}$ e criar velocidades de escapamento desejadas, por meio de um acelerador linear (MURRAY).

\subsection{POSTULADO DA RELATIVIDADE}

As leis da Física são as mesmas para os observadores em todos os referenciais inerciais. Não existe referencial inercial privilegiado.

Referencial inercial é aquele não sujeito a aceleração e que estiver em movimento com velocidade uniforme.

A explicação da gravitação levou Einstein à relatividade generalizada, que veremos a seguir. Tratase de estender ou generalizar o princípio da relatividade para aplicá-lo a sistemas acelerados, ou seja, não inerciais, além dos que se movem com velocidade uniforme. Com isso se pode resolver simultaneamente o problema da gravitação.

\subsection{TRANSFORMAÇÃO DE LORENTZ}

Como já mencionado, Einstein admitiu a velocidade da luz como um invariante físico, com o mesmo valor para todos os observadores. Essa exigência deve-se à aplicação da relatividade ao eletromagnetismo. Ver lei de Ampère-Maxwell na Seção 5.16, Equação 5, que na forma gaussiana, torna-se:

$$
{ }_{17} \text { Se } p=\frac{m_{0} v}{\sqrt{1-\beta^{2}}} \text { e } E=\frac{m_{0} c^{2}}{\sqrt{1-\beta^{2}}} \text { então } p=\frac{E v}{c^{2}} \text { e quando } v=c \Rightarrow, p=\frac{E}{c}=\frac{h \ni}{c}=\frac{h}{\lambda}
$$

Pois $c=\nabla \lambda(3)$

Também podemos notar que partículas que se propagam com a velocidade da luz, o tempo se torna nulo, ver Seção 7.2, Equações (1a) e (2). Quando $v=c, \beta=1, d t^{\prime}=0 \therefore t_{1}^{\prime}=t_{2}^{\prime}, t^{\prime}$ não varia. (4) 
$c^{2} \operatorname{rot} \vec{B}=\frac{\partial \vec{E}}{\partial t}+4 \pi \rho \vec{v}$, ver Seção 5.25.2, Equação 5.5b, quando falamos do tensor do campo eletromagnético. Podemos observar que $c$ participa da fórmula.

Sabemos que: $c^{2}=1 / \varepsilon_{0} \mu_{0}$ é a relação entre a velocidade da luz e as constantes dielétrica $\varepsilon_{0}$ e de permeabilidade $\mu_{0}$. Essa relação foi deduzida na Seção 5.23, Equação 5, sobre oscilações eletromagnéticas.

Com a invariância de $c$, a transformação de Galileu não pode ser correta (a não ser para baixas velocidades como $v<<c$. E, como já visto, o tempo não é absoluto).

Levando em consideração a Figura da Seção 1.3, vamos admitir que os observadores $O$ e $O^{\prime}$ estejam em movimento com velocidade relativa $v$, que os eixos $X$ e $X^{\prime}$ estejam dirigidos ao longo do movimento relativo e que os eixos $Y Y^{\prime}$ e $Z Z^{\prime}$ permaneçam paralelos. Admitimos que os observadores acertem seus relógios quando estiverem em coincidência, $t=t^{\prime}=0$, no instante inicial.

No instante $t=0$, um sinal luminoso é emitido da posição comum dos dois observadores. Após um intervalo de tempo $t$, o observador $O$ nota que a luz alcança o ponto A e anota $r=c t$.

Como: $r^{2}=x^{2}+y^{2}+z^{2}$ ou $x^{2}+y^{2}+z^{2}=c^{2} t^{2}$

Da mesma forma $O^{\prime}$ anota $r^{\prime}=c t^{\prime}$

$$
\therefore x^{\prime 2}+y^{\prime 2}+z^{\prime 2}=c^{2} t^{\prime 2}
$$

Temos que relacionar essas duas equações.

$$
y=y^{\prime} \text { e } z=z^{\prime} \quad x=v t \quad \text { ou } \quad x-v t=0
$$

Então $x^{\prime}=k(x-v t)$, com $k$ a ser determinado.

Da mesma forma: $\mathrm{t}^{\prime}=\mathrm{a}(\mathrm{t}-\mathrm{bx})$, em que $a$ e $b$ são constantes a serem determinadas.

Quando $k=a=1$ e $b=0$, teremos a transformação de Galileu: $\mathrm{t}^{\prime}=\mathrm{t}$.

Fazendo as substituições na Equação (2), obtemos:

$$
\begin{gathered}
k^{2}\left(x^{2}-2 v x t+v^{2} t^{2}\right)+y^{2}+z^{2}=c^{2} a^{2}\left(t^{2}-2 b x t+b^{2} x^{2}\right) \\
\therefore\left(k^{2}-b^{2} a^{2} c^{2}\right) x^{2}-2\left(k^{2} v-b a^{2} c^{2}\right) x t+y^{2}+z^{2}=\left(a^{2}-k^{2} v^{2} / c^{2}\right) c^{2} t^{2}
\end{gathered}
$$

Comparando com a Equação (1), obtemos o sistema de equações:

$$
\left\{\begin{array}{l}
k^{2}-b^{2} a^{2} c^{2}=1 \quad \text { Fazendo: } k^{2}=p, b^{2}=q, a^{2}=r \\
k^{2} v-b a^{2} c^{2}=0 \\
a^{2}-k^{2} v^{2} / c^{2}=1
\end{array}\right.
$$




$$
\begin{aligned}
& \left\{\begin{array} { l } 
{ p - c ^ { 2 } q r = 1 } \\
{ p v - r c ^ { 2 } \sqrt { q } = 0 } \\
{ r - ( v ^ { 2 } / c ^ { 2 } ) p = 1 }
\end{array} \quad \therefore \left\{\begin{array}{l}
p-\left[1-\left(v^{2} / c^{2}\right) p\right] c^{2} q=1 \\
p v-r c^{2} \sqrt{q}=0 \\
r=1+\left(v^{2} / c^{2}\right) p
\end{array}\right.\right. \\
& \left\{\begin{array} { l } 
{ p - c ^ { 2 } q - v ^ { 2 } p q = 1 } \\
{ v p - c ^ { 2 } \sqrt { q } [ 1 + ( v ^ { 2 } / c ^ { 2 } ) p ] = 0 }
\end{array} \quad \therefore \left\{\begin{array}{l}
\left(1-v^{2} q\right) p=1+c^{2} q \\
v p-c^{2} \sqrt{q}+v^{2} \sqrt{q} p=0
\end{array} \quad \therefore p=\frac{1+c^{2} q}{1-v^{2} q}\right.\right. \\
& v p(1-v \sqrt{q})=c^{2} \sqrt{q} \Rightarrow \frac{1+c^{2} b^{2}}{1-v^{2} b^{2}} v=\frac{c^{2} b}{1-v b} \Rightarrow \frac{\left(1+c^{2} b^{2}\right)}{c^{2} b}=\frac{1-v^{2} b^{2}}{1+v b} \\
& \frac{(1+v b)(1-v b)}{1-v b}=\frac{\left(1+c^{2} b^{2}\right) v}{c^{2} b} \Rightarrow c^{2} b+v e^{2} b^{2}=v+v c^{2} b^{2} \Rightarrow b=v / c^{2} \\
& p=\frac{1+c^{2}\left(v^{2} / c^{4}\right)}{1-v^{2}\left(v^{2} / c^{4}\right)}=\frac{1+v^{2} / c^{2}}{1-v^{4} / c^{4}}=\frac{1+v^{2} / c^{2}}{\left(1+y^{2} / c^{2}\right)\left(1-v^{2} / c^{2}\right)}=\frac{1}{1-v^{2} / c^{2}} \\
& \therefore k=\frac{1}{\sqrt{1-v^{2} / c^{2}}}=\gamma \quad r=1+\frac{1}{1-v^{2} / c^{2}} v^{2} / c^{2}=\frac{1-v^{2} / c^{2}+v^{2} / c^{2}}{1-v^{2} / c^{2}} \\
& \therefore a=\frac{1}{\sqrt{1-v^{2} / c^{2}}}=k=\gamma
\end{aligned}
$$

Assim, obtemos:

$$
\left\{\begin{array}{l}
x^{\prime}=k(x-v t)=\frac{x-v t}{\sqrt{1-v^{2} / c^{2}}}=\gamma(x-v t) \\
y^{\prime}=y \quad z^{\prime}=z \quad t^{\prime}=k(t-b x)=\frac{t-v x / c^{2}}{\sqrt{1-v^{2} / c^{2}}}=\gamma\left(t-v x / c^{2}\right)
\end{array}\right.
$$

São as transformações de Lorentz. 
Essa fórmula é geral, mas vale também para velocidades compatíveis com a da luz. Como caso particular, se fizermos $v$ muito menor que o da luz: $v<<c$, obtemos:

$$
x^{\prime}=x-v \cdot t \quad y^{\prime}=y \quad z^{\prime}=z \quad t^{\prime}=t
$$

Que são as transformações de Galileu, como visto na Seção 1.3.

Da equação (1) obtemos: $x^{\prime 2}-c^{2} t^{\prime 2}=x^{2}-c^{2} t^{2}$.

Essa fórmula sugere que $x, y, z$,ict $(i=\sqrt{-1})$ são interpretáveis (conforme MINKOWSKI) como coordenadas em um espaço quadridimensional, em que $x^{2}+y^{2}+z^{2}+(i c t)^{2}$ representa o quadrado da distância à origem. A ideia de Minkowski levou à concepção geométrica das leis fundamentais da física, que culminou com a inclusão da gravitação na chamada teoria da relatividade geral de Einstein.

Fisicamente, a última equação exprime que $x=c t$ implica $x^{\prime}=c t^{\prime}$, ou que a velocidade da luz é independente do movimento do observador. Essa é a explicação do resultado negativo da experiência de Michelson-Morley (BORN).

\subsection{TRANSFORMAÇÃO DE VELOCIDADES}

\subsubsection{Introdução}

A velocidade de $A$ medida por $O$ tem componentes:

$$
V_{x}=\frac{d x}{d t} \quad V_{y}=\frac{d y}{d t} \quad V_{z}=\frac{d z}{d t}
$$

Da mesma forma, $A$ medida por $O^{\prime}$ :

$$
V_{x}^{\prime}=\frac{d x^{\prime}}{d t^{\prime}} \quad V_{y}^{\prime}=\frac{d y^{\prime}}{d t^{\prime}} \quad V_{z}^{\prime}=\frac{d z^{\prime}}{d t^{\prime}}
$$

Nota-se que usamos $d t^{\prime}$ e não $d t$, pois $t$ e $t^{\prime}$ nesse caso não são iguais.

Diferenciando as equações (3), obtemos:

$$
d x^{\prime}=\frac{d x-v d t}{\sqrt{1-v^{2} / c^{2}}}=\frac{V_{x}-v}{\sqrt{1-v^{2} / c^{2}}} d t=\gamma\left(V_{x}-v\right) d t
$$

$d y^{\prime}=d y$

$d z^{\prime}=d z$

$$
d t^{\prime}=\frac{d t-v d x / c^{2}}{\sqrt{1-v^{2} / c^{2}}}=\frac{1-v V_{x} / c^{2}}{\sqrt{1-v^{2} / c^{2}}} d t=\gamma\left(1-v V_{x} / c^{2}\right) d t
$$


Substituindo $d x$ por $V_{x} d t$, dividindo as três primeiras equações pela quarta, obtemos:

$$
V_{x^{\prime}}^{\prime}=\frac{V_{x}-v}{1-v V_{x} / c^{2}} \quad V_{y^{\prime}}^{\prime}=\frac{V_{y}}{\gamma\left(1-v V_{x} / c^{2}\right)} \quad V_{z^{\prime}}^{\prime}=\frac{V_{z}}{\gamma\left(1-v V_{x} / c^{2}\right)}
$$

Constitui a lei de Lorentz para a transformação de velocidades, mais geral que a respectiva regra galileana.

Em particular, fazendo $v<<c$, isto é, para baixas velocidades $v$ muito inferiores a $c$, obtemos:

$$
V_{x^{\prime}}^{\prime}=V_{x}-v \quad V_{y^{\prime}}^{\prime}=V_{y} \quad V_{z^{\prime}}^{\prime}=V_{z}
$$

Que é a regra galileana para a comparação das velocidades de um corpo medidas por dois observadores em movimento relativo de translação

(ALONSO, FINN).

\subsection{DA VALIDADE DAS FÓRMULAS}

\subsubsection{Introdução}

Como a velocidade da luz é muito alta no vácuo, cerca de $300.000 \mathrm{~km} / \mathrm{s}$, é natural pensar que sua propagação fosse instantânea.

O primeiro a intuir de que seu valor fosse definido foi Galileu, que tentou medi-la colocando-se a certa distância de um ajudante, cada um com uma lanterna que poderia ser coberta e descoberta. Inicialmente, ele descobriu sua lanterna. Quando a luz foi vista pelo assistente, este imediatamente descobriu a sua, tornando-a visível para Galileu.

Para uma distância de $1 \mathrm{~km}$, sabe-se agora que o tempo de percurso é de apenas $7 \times 10^{-6} \mathrm{seg}$. Como é muito menor que o tempo de reação de uma pessoa, o método não serve.

Em 1675, Olaf Roemer, astrônomo dinamarquês, realizou observações dos satélites de Júpiter, pelo qual deduziu que a velocidade da luz fosse de $200.000 \mathrm{~km} / \mathrm{seg}$.

Cerca de 50 anos depois, James Bradley, astrônomo inglês, valendo-se da aberração da luz, em 1728, conseguiu um valor melhor: $304.000 \mathrm{~km} / \mathrm{seg}$.

Em 1849, Fizeau (1819-1896), físico francês, utilizando uma roda dentada e observando a interferência das imagens em um espelho semiprateado, obteve: $313.300 \mathrm{~km} / \mathrm{seg}$.

Albert Abraham Michelson utilizou um espelho rotatório em vez da roda dentada. O valor conseguido em 1880 foi de $299.910 \mathrm{~km} / \mathrm{seg}$. 
As medições mais atualizadas, por meio do geodímetro atestam: $299.792,9 \mathrm{~km} / \mathrm{seg}$.

Tendo em vista esse valor, é compreensível que para os fenômenos usuais na Terra, onde a maior velocidade conseguida por um foguete foi cerca de $10 \mathrm{~km} / \mathrm{seg}$, não se conseguisse detectar nenhuma alteração nos conceitos clássicos, em que as fórmulas são válidas, pois $30 / 300.000=0,0001$, ou seja aproximadamente $0,01 \%$ da velocidade da luz. Usando as fórmulas relativísticas, vimos que se obtêm as fórmulas usuais de Galileu.

Assim como a velocidade da luz, também era usual pensar, como Newton, que a propagação das interações gravitacionais fosse instantânea. ${ }^{18}$

Porém, nos fenômenos atômicos, em que temos velocidades compatíveis com a da luz, as fórmulas clássicas falham, sendo forçoso levar em consideração os efeitos relativísticos.

Também, em Astronomia, observamos que a luz leva cerca de $1 \mathrm{seg}$ para chegar da Lua, e aproximadamente $8 \mathrm{~min}$, do Sol. Em casos de estrelas, a mais próxima, Alfa Centauri, se encontra a 4 anos-luz (o tempo que sua luz leva para chegar a nós), e a galáxia mais próxima, a Grande Nuvem de Magalhães, encontra-se a cerca de 180.000 anos-luz.

Então, a imagem que vemos não é atual, ela é do passado. Por isso, é impossível se falar em tempo absoluto, pois não sabemos o que está ocorrendo agora por lá. Fica, pois evidente a existência de uma $4^{\mathrm{a}}$ dimensão, o tempo.

O tempo só pode ser considerado imutável para fenômenos locais na Terra, a baixas velocidades, onde se podem aplicar as fórmulas clássicas. Nos outros casos, devemos levar em conta os efeitos relativísticos, função da velocidade do objeto em questão e sua relação com a velocidade da luz, isto é: $\beta=v / c$ e o consequente fator de Lorentz: $\gamma=1 / \sqrt{1-\beta^{2}}$

Conclui-se que as interações se propagam com a velocidade da luz, incluindo as interações gravitacionais onde são mais notadamente observáveis. Não são, portanto, instantâneas. (1)

Vale a pena salientar que, com base na observação do final da Seção 7.9.1 (Equação 4, Exemplos ilustrativos da Seção 7.8), se a velocidade do fóton é igual à da luz, o fóton em si não percebe a passagem do tempo e, nesse sentido, para o fóton, a propagação é instantânea.

\subsection{DECAIMENTO RADIOATIVO, MEIA-VIDA}

Expressando a natureza estatística do processo de decaimento em uma amostra contendo $\mathrm{N}$ núcleos radioativos, a taxa de decaimento é: $-\mathrm{dN} / \mathrm{dt}$, proporcional a $\mathrm{N}:-\frac{\mathrm{dN}}{\mathrm{dt}}=\lambda \mathrm{N}$, sendo $\lambda$ a constante de desintegração, com valor característico para cada radionuclídeo.

\footnotetext{
${ }^{18}$ Na verdade, Newton não associava a interação gravitacional com a propagação da luz, como o fazemos na atualidade.
} 
Integrando na forma:

$$
\int_{\mathrm{N}_{0}}^{\mathrm{N}} \frac{\mathrm{dN}}{\mathrm{N}}=-\int_{0}^{\mathrm{t}} \lambda \mathrm{dt} \quad \therefore \ln \frac{\mathrm{N}}{\mathrm{N}_{0}}=-\lambda \mathrm{t} \Rightarrow \mathrm{N}=\mathrm{N}_{0} \mathrm{e}^{-\lambda \mathrm{t}}=\mathrm{N}_{0} \exp (-\lambda \mathrm{t})
$$

Recorde-se que: $10^{2}=100 \Rightarrow \log _{10} 100=2 \Rightarrow b=e^{a}=\exp (a) \Rightarrow \log _{e} b=\ell n b=a^{19}$

Mostrando que o número de núcleos sobreviventes decresce exponencialmente.

Definindo a "meia-vida" $\mathrm{T}$ como o intervalo de tempo no qual $\mathrm{N}$ se reduz à metade do valor inicial: $1 / 2 \mathrm{~N}_{0}=\mathrm{N}_{0} \mathrm{e}^{-\lambda \mathrm{t}}$

$$
\begin{aligned}
& \left.\begin{array}{l}
\mathrm{N}_{1}=\mathrm{N}_{0} \exp \left(-\lambda \mathrm{t}_{1}\right) \\
\mathrm{N}_{2}=\mathrm{N}_{0} \exp \left(-\lambda \mathrm{t}_{2}\right)
\end{array}\right\} \Rightarrow \frac{\mathrm{N}_{1}}{\mathrm{~N}_{2}}=\exp \left[\lambda\left(\mathrm{t}_{2}-\mathrm{t}_{1}\right)\right]=2 \Rightarrow \ell \mathrm{n} 2=\lambda\left(\mathrm{t}_{2}-\mathrm{t}_{1}\right) \\
& \therefore \frac{\ln 2}{\lambda}=\mathrm{t}_{2}-\mathrm{t}_{1}=\mathrm{T} \quad \text { Logo: } \mathrm{T}=\frac{0,693}{\lambda}
\end{aligned}
$$

Exemplo: seja uma amostra de Iodo $128: \mathrm{I}^{128} \mathrm{com}$ as seguintes taxas de desintegração evoluindo no tempo.

$$
\begin{gathered}
\ell \mathrm{n} \frac{\mathrm{N}}{\mathrm{N}_{0}}=\ell \mathrm{nN}-\ell \mathrm{nN}_{0}=-\lambda \mathrm{T} \\
\operatorname{lnN}=\operatorname{lnN}_{0}-\lambda \mathrm{T} \\
\therefore-\lambda=\frac{6,2-0}{225 \mathrm{~min}-0}, \text { inclinação da reta } \quad \therefore \lambda=0,0275 \mathrm{~min}^{-1}
\end{gathered}
$$

Sua meia-vida é:

$$
\mathrm{T}=\frac{0,693}{\lambda}=\frac{0,693}{0,0275 \mathrm{~min}^{-1}} \cong 25 \mathrm{~min}
$$

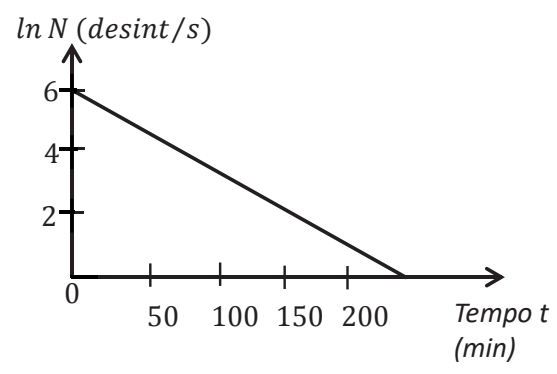

9 * Série aritmética: 12345 , logarítmo com base de 32:5 - $\log 32=5$

Série geométrica: 2481632 , antilog com base 2 de 5:32: 2 (elevado a quinta) =32 
Trata-se de um exemplo de equação logarítmica e também da fração exponencial. Serve para calcular qual será o valor do capital ao final de certo tempo t, quando o capital inicial for $y_{0}$ e com juros $\alpha=1 / 100$ , sendo i a porcentagem: $\mathrm{y}=\mathrm{y}_{0} \mathrm{e}^{\alpha \mathrm{t}}$. Nesse caso, a função em vez de ser decrescente, será crescente, por isso o sinal positivo de $\alpha$, em lugar de ser negativo (COURANT). Recorde-se que o valor e é determinado com o seguinte lim: $\mathrm{e}=\lim _{n \rightarrow \infty}\left(1+\frac{1}{n}\right)^{n}$ (GRANVILLE) ver secção 7.2, equação $1 \mathrm{~A}$.

O iodo radioativo é utilizado para diagnóstico da tireoide, e como sua quantidade é muito pequena, não causa danos ao organismo.

Exemplo: 46,3mg de potássio mostra uma atividade por partículas $\beta$ (elétrons) de 1,5 desint/s. O isótopo responsável é $\mathrm{K}^{40}$, que se encontra na concentração até $0,012 \%$ na mistura natural. Calcular a meia vida do $\mathrm{K}^{40}$.

Quantidade de átomos $\mathrm{K}^{40} \mathrm{em} 46,3 \mathrm{mg}$ de potássio natural:

$$
\mathrm{N}=\frac{0,012}{100} \times 46,3 \times 10^{-3} \mathrm{~g} \times \frac{6,02 \times 10^{23} \mathrm{at} / \mathrm{mol}}{40^{\mathrm{g}} / \mathrm{mol}} \text {, em que } \mathrm{A}=6,02 \times 10^{23} \mathrm{at} / \mathrm{mol} \text {, é o número de Avogadro. }
$$

(ver Secção 7.6).

$$
\begin{gathered}
\text { Portanto: } \mathrm{N}=8,37 \times 10^{16} \text { átomos e } \mathrm{T}=\frac{0,693}{\lambda}=\frac{0,693 \mathrm{~N}}{\mathrm{dN} / \mathrm{dt}}=\frac{0,693 \times 8,37 \times 10^{16} \text { át }}{1,5^{\mathrm{desint} / \mathrm{s}}}= \\
=3,87 \times 10^{16} \mathrm{~s}=1,23 \times 10^{9} \text { anos. }
\end{gathered}
$$

Esse tempo é comparável com o início da vida estimada na Terra. Não estranhe o fato de não ser possível medir a meia-vida desse radionuclídeo, esperando que sua taxa de decaimento diminua. Deve-se mencionar que o potássio presente em nosso organismo tem uma parcela normal desse radioisótopo. Todos nós somos levemente radioativos!

Um teorema simples define a função exponencial.

Se a função $\mathrm{y}=\mathrm{f}(\mathrm{x})$ satisfizer a equação diferencial: $\mathrm{y}^{\prime}=\alpha \mathrm{y}$, em que $\alpha$ é uma constante $\neq 0$, isto é, diferente de zero, $\mathrm{y}$ toma a forma: $\mathrm{y}=\mathrm{f}(\mathrm{x})=\mathrm{c} \mathrm{e}^{\alpha \mathrm{x}}$, em que c é também uma constante. Inversamente, cada função, expressa como $c \mathrm{e}^{\alpha \mathrm{x}}$, satisfaz a equação $\mathrm{y}^{\prime}=\alpha \mathrm{y}$.

Por exemplo, no caso do decaimento radioativo, teremos: $y=-\frac{d N}{d t}=\lambda N$, consequentemente: $N=$ $\mathrm{N}_{0} \mathrm{e}^{-\lambda \mathrm{t}}$

Sabendo que "desintegração" é a perda de massa por meio da emissão de partículas radioativas, para uma massa de um isótopo de rádio Ra, as experiências mostram que $\lambda=3,85 \times 10^{-3} \mathrm{~s}^{-1}$. Achar a meia vida $\mathrm{T}$ desse isótopo, isto é, o tempo necessário para que a massa da substância decaia à metade do que era.

$$
\begin{gathered}
\text { Então: } 1 / 2 \mathrm{~N}_{0}=\mathrm{N}_{0} \exp (-0,00385 \mathrm{~T}) \\
\Rightarrow \ln ^{2-1}=-0,00385 \mathrm{~T} \Rightarrow-\ln ^{2}=\ln \mathrm{e}^{-0,00385 \mathrm{~T}} \\
\therefore \mathrm{T}=\frac{\ln 2}{0,00385} \simeq 180 \mathrm{~s}
\end{gathered}
$$


Logo, a meia-vida desse isótopo de Ra é de cerca de 3min. Perceba que é muito mais fácil tratar da meia-vida do que sobre a constante $\lambda$, pois é muito mais fácil falar "três minutos" do que "três virgula oitenta e cinco vezes dez elevado a menos três". Isso justifica o emprego do conceito meia-vida (LIVERHANT; HALLIDAY, RESNICK, WALKER, COURANT, GRANVILLE, KAPLAN, THOMPSON). ${ }^{20}$

\subsection{UMA FORMA SIMPLES DE DEDUZIR A FÓRMULA DE EINSTEIN DA RELATIVIDADE RESTRITA OU ESPECIAL: $\mathrm{E}_{\mathbf{c}}=(\Delta \mathbf{m}) \mathbf{c}^{2}$}

Maxwell deduziu que a luz é onda eletromagnética, comprovado posteriormente por Hertz. Trinta anos mais tarde, Nichols e Hull nos Estados Unidos, e Lebedev na Rússia, em 1903, mediram a denominada pressão de radiação (HALIDAY, RESNICK, WALKER), ${ }^{21}$ que é a variação da quantidade de movimento a qual é função da energia da onda eletromagnética. Como sabemos, o movimento ondulatório transporta energia de um local para outro, por exemplo, o som transportado através do ar ou a radiação solar, transportada do Sol até a Terra através do que Maxwell acreditava ser o "éter luminífero". 22

\section{De fato:}

$\mathrm{dE}_{\mathrm{c}}=\mathrm{Fdx}, \mathrm{F}=\mathrm{m} \frac{\mathrm{dv}}{\mathrm{dt}}, \mathrm{dE}_{\mathrm{c}}=\operatorname{mvdv}, \mathrm{E}_{\mathrm{c}}=\frac{1}{2} \mathrm{mv}^{2}=\frac{1}{2} \mathrm{pv}$ pois: $\mathrm{p}=\mathrm{mv}$ (quantidade de movimento) $\Rightarrow$ $\mathrm{p}=2 \frac{\mathrm{E}_{\mathrm{c}}}{\mathrm{v}}$, relação encontrada por Maxwell, entre quantidade de movimento e energia cinética $\mathrm{E}_{\mathrm{c}}$.

Recordemos: quando um jato d'água incide em uma superfície vertical, a variação da quantidade de movimento será dada por: $\mathrm{mv}-(-\mathrm{mv})=2 \mathrm{mv}$.

De modo semelhante, quando a luz incide em um espelho vertical, teremos: $\mathrm{P}_{\text {luz }}=2 \mathrm{mc}$, que é variação da quantidade de movimento, supondo reflexão total, se houver absorção, $\mathrm{P}_{\text {luz }}=\mathrm{mc}$. Comparando com a expressão de p, obtemos: $\frac{2 \mathrm{E}_{\mathrm{c}}}{\mathrm{c}}=2 \mathrm{mc} \Rightarrow \mathrm{E}_{\mathrm{c}}=\mathrm{mc}^{2}$, a famosa equação da Relatividade especial, deduzida por Einstein. Na verdade, é: $\mathrm{E}_{\mathrm{c}}=(\Delta \mathrm{m}) \mathrm{c}^{2}$, pois se trata de variação da massa.

Posteriormente, descobriu-se que as pequenas diferenças de massa, antes e depois das reações nucleares, denominada energia de ligação das partículas nucleares, são responsáveis pela grande liberação de energia nos processos dos elementos radioativos (os elementos descobertos por Marie Curie e outros). Como exemplo, temos a descoberta da fissão nuclear do urânio por Lisa Meitner e Otto Frisch, cujo isótopo $\mathrm{U}_{235}$ pode resultar em reação em cadeia, resultando na bomba atômica e nas usinas nucleares. Também as reações de fusão de $\mathrm{H}$ para formar $\mathrm{He}$, que fornecem a energia liberada pelas estrelas, como o Sol, e na bomba de H.

${ }^{20}$ Silvanus Thompson em Calculus Made Easy, o livro teve tanto sucesso que está na internet, em domínio público.

${ }^{21}$ Média das medições efetuadas com balança de torção: $7,01 \mu \mathrm{N} / \mathrm{m}^{2}$, com previsão de $7,05 \mu \mathrm{N} / \mathrm{m}^{2}$, medidos em unidades de pressão.

${ }^{22} \mathrm{Na}$ atualidade, substituímos o éter luminífero pelo conceito do campo eletromagnético (GAMOW).

Observação: pressão é a energia por unidade de volume.

Como $m v=F \Delta t=$ impulsão

Conhecendo a área onde a pressão é aplicada, obtemos a força $F$ e, sabendo o intervalo de tempo em que ela é aplicada, obtemos a quantidade de movimento $m v$. 
Reação termonuclear "próton-próton":

$4{ }_{1} \mathrm{H}^{1} \rightarrow{ }_{2} \mathrm{He}^{4}+2 \beta^{+}+2 \gamma+2 \gamma+26,7 \mathrm{Mev}$

$\beta^{+}$partículas $\beta$ de pósitrons

$\gamma$ raios $\gamma$

$\checkmark$ neutrinos

26,7 Mev - energia liberada em megaeletronvolts.

Ciclo de Bethe ou de carbono-nitrogênio:

Ocorre com a liberação de cerca $26 \mathrm{Mev}$ proposto por Hans Bethe em 1939, ver a figura.

Pr são prótons, núcleos de ${ }_{1} \mathrm{H}^{1}$.

Por alguns anos se pensou que o ciclo C-N fosse responsável pela produção de quase toda energia solar, porém, de acordo com dados recentes, a cadeia próton é agora considerada mais importante do que o ciclo C. Pensa-se que o ciclo C produza mais energia nas estrelas da sequencia principal muito mais luminosas do que o Sol e cujas temperaturas centrais são mais altas, enquanto a cadeia de próton é mais importante para as estrelas da sequencia principal menos luminosas do que o Sol (KAPLAN, GAMOW, KAHN, BETHE, GAMOW).

Ciclo Bethe

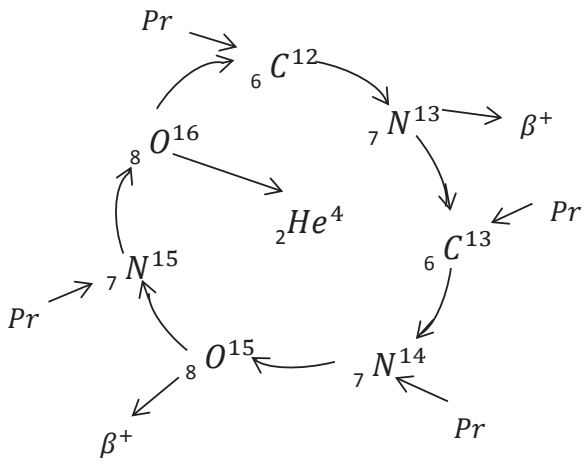

Para calcular a força, ver exemplo em (ROTH, 2012, Secção 5.1, cap. 5).

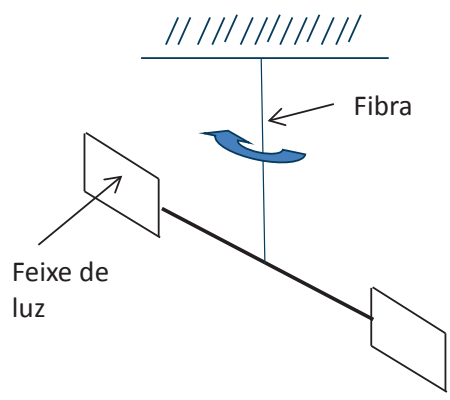

Balança de Torção usada por Nichols e Hull. Os detalhes da delicada experiência foram omitidos. 
Observemos que o éter luminífero era considerado uniformemente distribuído no espaço, e os campos elétricos e magnéticos têm um valor máximo na origem que diminui com a distância até se anular.

\subsubsection{Se fótons não têm massa, como podem ter quantidade de movimento?}

A teoria da relatividade especial apresenta para a energia: $\mathrm{E}^{2}=\left(\mathrm{m}_{0} \mathrm{c}^{2}\right)+(\mathrm{pc})^{2}$, em que $\mathrm{m}_{0}$ é a massa de repouso da partícula. A massa de repouso do fóton é nula, então: $\mathrm{E}=\mathrm{pc}=\mathrm{mc}^{2}, \operatorname{logo}: \mathrm{m}=\mathrm{p} / \mathrm{c}$, sendo $\mathrm{m}$, a massa relativística, que não é nula.

Conforme Broglie, estabelecendo a dualidade entre onda e partícula, o comprimento de onda: $\lambda=$ $\mathrm{h} / \mathrm{p}$, onde h é a constante de Planck. Então: $\mathrm{p}=\mathrm{h} / \lambda$. ver Introdução do anexo A6 secção A 6.1: $\mathrm{p}=\mathrm{E} / \mathrm{c}=$ $\mathrm{h} \nu / \mathrm{c}=\mathrm{h} / \lambda, \mathrm{c}=\lambda \nu$

Relacionando o comprimento de onda e a quantidade de movimento: $\mathrm{m}=\mathrm{E} / \mathrm{c}^{2}=\mathrm{h} / \lambda \mathrm{c}, \operatorname{logo}$, os fótons têm massa inversamente proporcional ao seu comprimento de onda.

Pela teoria de Newton, os fótons sofrem influência da gravidade. Einstein generalizou a massa newtoniana na massa relativística, podendo tratar as duas como se fossem a mesma coisa (UNIVERSITY OF TORONTO).

\subsubsection{Equivalência entre massa e energia}

Como visto anteriormente, em fins do século XIX, Maxwell deixou claro que a luz transporta quantidade de movimento. Com essa propriedade, Einstein sugeriu um raciocínio que, por meio do princípio da inércia, associa massa à energia.

Imagine-se uma caixa em repouso em referencial inercial. A radiação é emitida da parede à esquerda, veja-se figura adiante. Como existe conservação da quantidade de movimento, a caixa recua em sentido oposto. Quando a radiação for absorvida na parede à direita, a caixa para. Como não existem forças externas atuando na caixa, devemos admitir a existência de massa na energia da radiação, para não violar o princípio da inércia. Essa seria a massa relativística (FERRAZ NETTO, em feira de Ciências).

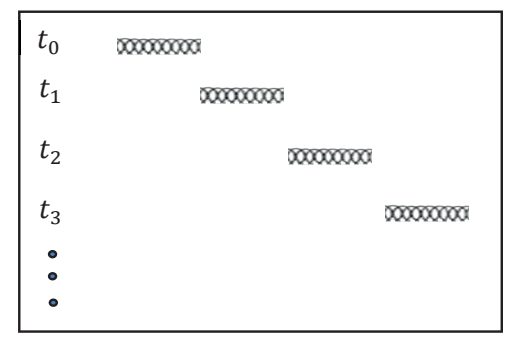

Em 1887, Hertz descobriu que uma superfície metálica pode emitir eletricidade quando a luz ultravioleta incide nela e, em 1898, Thomson mostrou que o valor de e/m das partículas emitidas é o mesmo que o dos raios catódicos. Agora sabemos que essas partículas são elétrons, no que ficou conhecido 
como efeito fotoelétrico. Esse fenômeno não pode ser explicado se considerarmos a luz como de natureza ondulatória.

Em 1905, Einstein introduziu o conceito de fóton e deu uma explicação quantitativa desse efeito.

Em consequência do princípio da conservação de energia, cada fóton deve dar toda energia $h$ ฟ a um elétron. Para que o elétron saia do metal, ele deve gastar energia contra a barreira de potencial do metal, chamada função de trabalho $\phi$. A equação da energia cinética máxima dos elétrons emitidos será: $1 / 2 m v_{m}^{2}=h \gamma-\phi$, sendo $v_{m}$ a velocidade máxima dos elétrons.

Então deduzimos ser o fóton partícula que cede sua energia cinética aos elétrons.

O efeito Compton é outro fenômeno no qual raios X perdem energia ao colidir com elétrons. Foi interpretado por Compton como se partículas de raios X colidissem com elétrons, como bolas de bilhar, desviando-se e provocando movimentação desses elétrons.

Um fóton de frequência $\gamma$, tem energia $h \gamma$, quantidade de movimento $h \bigvee / c$.

Após o impacto com um elétron livre, produz-se um fóton espalhado de frequência $\gamma^{\prime}$, menor do que $\gamma$ e um elétron de recuo. A energia e a quantidade de movimento do fóton incidente antes do impacto são iguais à energia e quantidade de movimento do fóton espalhado e do elétron de recuo após o impacto.

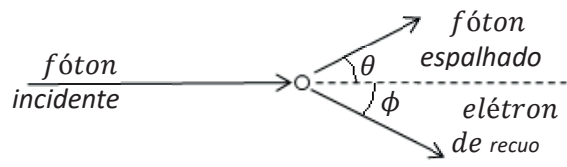

Logo a quantidade de movimento do fóton $\left.\quad(h)^{\prime} \operatorname{sen} \theta\right) / c$ deve ser igual à quantidade de movimento do elétron de recuo vezes sen $\phi$, são perpendiculares à trajetória do fóton incidente e de sentidos opostos entre si.

No entanto, quando o fóton tiver energia maior do que 2 vezes a energia de repouso do elétron, o fóton pode desaparecer e produzir duas partículas: um elétron e um pósitron, isto é, um elétron positivo.

Nota-se que: $\mathrm{e}^{-}+\mathrm{e}^{+} \rightarrow \gamma+\gamma \quad(\mathrm{Q}=1,02 \mathrm{Mev})$, e cada fóton $\gamma$ tem $0,51 \mathrm{Mev}$ de energia. Essa é a reação de aniquilação do pósitron, é o inverso da formação do par elétron-positron à custa dos raios $\gamma$ (FERENCE e LEMON, KAPLAN).

Os elementos químicos são formados nos núcleos das estrelas. São indispensáveis em nossa estrutura física. Os átomos de $\mathrm{O}$ que respiramos, o Ca dos nossos ossos, e o $\mathrm{Fe}$ e o $\mathrm{C}$ da musculatura tiveram uma origem cuja história agora conhecemos. $\mathrm{H}, \mathrm{He}$, deutério e parte do Li, foram formados no Big Bang. Os demais, incluindo o He foram sintetizados no centro das estrelas. Após a morte das estrelas, o gás enriquecido com esses elementos é lançado ao espaço, juntando-se ao de outras estrelas. Forma-se uma nova geração de corpos celestes. O Sol é uma estrela de $3^{a}$ geração, por isso, a composição do sistema solar é suficientemente rica para a vida como conhecemos (STEINER). 


\subsubsection{Exemplo de aplicação}

\subsection{Sintese de elementos pesados}

A nucleossíntese de elementos leves como: $\mathrm{Ne}, \mathrm{O}, \mathrm{Si}$ etc. até o $\mathrm{Fe}^{26}$ são produzidos por fusão em reações exoenergéticas, isto é, com liberação de energia. Os mais pesados que o Fe, como $\mathrm{Au}, \mathrm{Hg}, \mathrm{Pb}, \mathrm{Ni}, \mathrm{U}, \mathrm{Th}$ etc. são sintetizados por reações endotérmicas, isto é, que absorvem parte da energia armazenada pela estrela com as reações exoenergéticas. São formadas na exposição dos núcleos leves ao fluxo de nêutrons, em temperaturas moderadas. Os nêutrons, por serem neutros, não necessitam vencer a barreira coulombiana de íons. Reação típica: $\mathrm{C}^{13}(\alpha, n) \mathrm{O}^{16}(0)$. O íon capturando um nêutron se torna um isótopo do mesmo elemento, com uma unidade a mais de massa atômica: $(\mathrm{Z}, \mathrm{A})+\mathrm{n} \rightarrow(\mathrm{Z}, \mathrm{A}+1)+\gamma$.

Se o núcleo $(\mathrm{Z}, \mathrm{A}+1)$ for estável, poderá capturar outro nêutron antes ou depois do decaimento $\beta$. Tem-se então o processo slow, s, antes do decaimento $\beta$, ou o processo rapid, r, decaimento $\beta$ antes da captura de um novo nêutron. Essa nomenclatura foi introduzida por Eleanor Margaret Peachey Burbidge (1919), Geoffrey Ronald Burbidge (1925-2010), William Alfred Fowler (1911-1995) e Sir Fred Hoyle (1915-2001) (SOUZA, SARAIVA, UFRGS, 2000).

\subsection{RELAÇÃO ENTRE RELATIVIDADE ESPECIAL E FÍSICA NUCLEAR}

\subsubsection{Henri Becquerel e experiências de Rutherford ${ }^{23}$}

Esse eminente físico francês (1852-1908), ao examinar os sais de um metal raro, o urânio, descobriu que emitiam um raio aparentemente capaz de penetrar os objetos opacos. Um composto de urânio, que ele colocou sobre uma chapa fotográfica envolvida em papel negro, tinha impressionado a chapa "através" do papel. Isso, ao que nos consta, foi a primeira vez que um homem observou as estranhas propriedades penetradoras de certos raios.

No início de 1896, Becquerel realizou experiências com sal duplo de U, sabendo ser de elevada fluorescência. Colocou cristal sobre a chapa fotográfica, envolta em papel preto e expôs à luz do Sol. A luz induziria fluorescência e, se esta contivesse raios X, eles penetrariam o papel preto e registraria na chapa fotográfica.

No outono de 1895, Wilhelm Röntgen examinava fenômenos de luminescência. Passou corrente elétrica por um tubo de vácuo parcial (tubo de raios catódicos, semelhante aos usados em antigos aparelhos de TV).

A fim de examinar a débil luminescência, colocou o tubo dentro de uma caixa preta de papelão. Quando ligou a corrente, viu luminescência fora da caixa de papelão.

\footnotetext{
${ }^{23}$ Ernest Rutherford (1871-1937), físico neo-zelandês. Esse notável pesquisador foi orientador de Niels Bohr em Manchester. Foi agraciado com título de nobreza, como Primeiro Barão Rutherford de Nelson. Estava aguardando cirurgia de hérnia umbilical, mas como só poderia ser operado por um nobre (exigência do protocolo britânico), a demora custou-lhe a vida.
} 


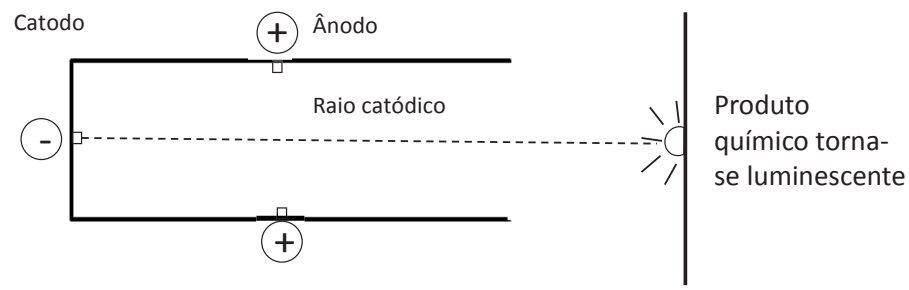

Descobriu que havia uma folha de papel revestida com camada de platinocianeto de Bário, produto luminescente. Como era possível, se o tubo estava encerrado na caixa preta? Não sabendo a natureza da radiação, chamou-a de raio $\mathrm{X}$.

No caso do cristal de U, Becquerel pensou tratar-se de fenômeno parecido com o raio X e que alguma radiação invisível tivesse energizado o cristal.

Seguindo o mesmo caminho de Becquerel, Marie e Pierre Curie investigaram compostos de $\mathrm{U}$, chegando na pechblenda, um minério betuminoso marrom escuro, mineral de óxido de $\mathrm{U}$, altamente radioativo. Com o efeito piezoelétrico, descoberto por Pierre e seu irmão Jacques, mediram a corrente elétrica induzida no ar ionizado pela radioatividade.

No caso da pechblenda, mediu-se $83 \times 10^{-12} \mathrm{~A}$, enquanto alguns sais de $\mathrm{U}$ registravam menos de $0,3 \times 10^{-12} \mathrm{~A}$.

Descobriu-se que o Tório exigia, para ser neutralizado, uma corrente de $53 \times 10^{-12} \mathrm{~A}$.

Concluiu que a pechblenda deveria conter outro elemento radioativo além do U.

Após exaustivas e trabalhosas operações de refinamento com minério de pechblenda, descobriram o polônio, em julho de 1898 , de número atômico 84 , contido em pó de bismuto ${ }^{24}$ e semelhante a este, seu número atômico seria determinado muito depois. O polônio é 400 vezes mais radioativo que o U.

No mesmo ano, isolaram um novo elemento no cloreto de bário ao qual deram o nome de rádio ${ }^{25}$, mais tarde associado ao número atômico 88 , cuja radioatividade é 3 milhões de vezes maior que a do U .

Pierre, Becquerel e Rutherford passaram a radiação por um campo magnético e descobriram três tipos: $\alpha, \beta$ e $\gamma$, assim denominados por Rutherford. Pierre descobriu que os raios $\beta$ têm carga negativa, posteriormente descobriu-se que são elétrons. Rutherford descobriu que os raios $\alpha$ são positivos, e posteriormente se verificou serem núcleos de He. Posteriormente, Rutherford descobriu que os raios $\gamma$ são neutros, e também são radiação eletromagnética de curto comprimento de onda e alta energia, isto é, de frequência alta.

\footnotetext{
24 Sulfeto de bismuto.

${ }^{25}$ Propriedades semelhantes às do bário.
} 
Em 1903, Becquerel, Pierre e Marie Curie dividiram o Prêmio Nobel de Física. Becquerel recebeu pela descoberta da radioatividade espontânea, e Pierre e Marie Curie pelas pesquisas sobre os fenômenos da radiação descoberto por Becquerel.

Antes deles, Wilhelm Röntgen foi o primeiro a receber Prêmio Nobel, em 1901, pela descoberta dos raios $\mathrm{X}$.

Pierre Curie descobriu que $1 \mathrm{~g}$ de Rádio emite 140 calorias/hora, o suficiente para ferver água. Escreveu em seu relatório: "Cada átomo de um corpo radioativo funciona como uma fonte constante de energia, o que implica uma revisão do Princípio de conservação de energia”. Quando a notícia veio a público, a imprensa estampou: "Os Curie descobrem o moto-perpétuo".

A radioatividade conduziu a ciência para uma nova era.

Desde a Antiguidade, quando Demócrito propôs a ideia de a matéria ser formada por "átomos”, que significam "indestrutíveis".

Em 1871, o químico siberiano Dmitri Mendeleiev praticamente reinventou a Química ao publicar a Tabela periódica dos Elementos. Listava todos os elementos químicos conforme seu peso atômico e valência, prevendo também elementos que ainda não tinham sido descobertos.

Enquanto os Curies investigavam em Paris, Rutherford e Soddy faziam o mesmo no Canadá. A abordagem deles é mais teórica, e em 1902 publicaram o artigo: “A causa e a natureza da radioatividade", no qual declaram que a diferença entre os raios X e a radioatividade, até então tidas como semelhantes, é que os raios $\mathrm{X}$ são produzidos quando uma substância é bombardeada, enquanto a radioatividade ocorre espontaneamente.

Concluíram ser a radioatividade um fenômeno atômico. Uma forma de decaimento atômico, na qual alguns átomos pesados, instáveis, se desintegram para se converter em átomos mais leves e mais estáveis. "As mudanças devem estar ocorrendo dentro do átomo." ${ }^{26}$

Os Curies demonstraram que a radioatividade era uma fonte colossal de energia.

Ninguém sabia o que estava acontecendo.

O $1^{\circ}$ passo aconteceu 2 anos mais tarde, quando um obscuro físico amador suíço ${ }^{27}$, trabalhando na Agência de Patentes de Berna, teve uma ideia. Em 1905, Albert Einstein publicou seu artigo sobre a relatividade especial, na qual derivou a famosa fórmula: $\mathrm{E}=\mathrm{mc}^{2}$.

Ou seja: uma diminuta quantidade de matéria, m, podia se transformar em uma quantidade colossal de energia em função da constante c (a velocidade da luz no vácuo). É isso que causa a radioatividade. O que Becquerel e os Curie descobriram, conduziria, um dia, à energia nuclear e às bombas atômicas (THOMAS, THOMAS, STRATHERN, STRATHERN, PAULING).

\footnotetext{
${ }^{26}$ Exemplificando: $\operatorname{ThC}\left(B i^{212}\right), 66,3 \%$ desintegra por partículas $\beta$ e 33,7\%, por emissão $\alpha$. ThC emite $\beta$ para formar $T h C^{\prime}\left(P o^{212}\right)$ ou $\alpha$ para formar $T h C^{\prime \prime}\left(T \ell^{208}\right)$; Th $C^{\prime}$ emite $\alpha$ para fornecer $P b^{208}$ estável, enquanto $T h C^{\prime \prime}$ emite $\beta$ para também formar $P b^{208}$ estável (KAPLAN).

27 Nascido na Alemanha. Na ocasião, ele tinha cidadania suíça.
} 


\subsubsection{Experiências de Rutherford}

Em 1919, antes de deixar Manchester para assumir a direção do laboratório Cavendish em Cambridge, Rutherford se tornou a primeira pessoa a transmutar um elemento em outro. Ele bombardeou nitrogênio com radiação $\alpha$, convertendo-o em oxigênio:

$$
{ }_{7} \mathrm{~N}^{14}+{ }_{2} \mathrm{He}^{4} \rightarrow\left[{ }_{9} \mathrm{~F}^{18}\right] \rightarrow{ }_{8} \mathrm{O}^{17}+{ }_{1} \mathrm{H}^{1}
$$

Com isso, identificou partículas de hidrogênio, ${ }_{1} \mathrm{H}^{1}$, demonstrando que elas são constituintes do núcleo de nitrogênio, N, e também de outros núcleos. Essa hipótese já havia sido levantada por Prout. ${ }^{28}$ Por esses motivos, em 1920, Rutherford postulou que o núcleo de $\mathrm{H}$ deveria ser uma partícula fundamental, que ele denominou de "próton", e que seria constituinte de todos os demais núcleos. Assim, agora Rutherford é considerado o fundador da Física Nuclear.

Em 1932, ele detectou, juntamente com Walton e Cockroft, a captura de um próton, ${ }_{1} \mathrm{H}^{1}$, pelo lítio 7, ${ }_{3} \mathrm{Li}^{7}$, decompondo-o em duas partículas $\alpha,{ }_{2} \mathrm{H}^{4}$, e liberando energia. Dois anos mais tarde, conseguiu, com Oliphant e Harteck, efetuar a fusão de dois dêuterons, ${ }_{1} \mathrm{H}^{2}$, que se transformaram em hélio $3,{ }_{2} \mathrm{He}^{3}$, e um nêutron, ou em trítio, ${ }_{1} \mathrm{H}^{3}$, e um próton, ${ }_{1} \mathrm{H}^{1}$, liberando energia em ambos.

Introduziu o conceito de núcleo atômico ao investigar a dispersão das partículas $\alpha$ por folhas delgadas de metal. Verificou que a grande maioria das partículas atravessava a folha sem se desviar e concluiu, com base nessas observações e em cálculos, que os átomos de ouro, Au, e por extensão, quaisquer outros, são estruturas praticamente vazias e não esferas maciças, como Thomson havia imaginado.

Observação: a massa atômica $\simeq$, isto é, aproximadamente igual, pois depende do número de nêutrons nos isótopos e da quantidade relativa dos isótopos, cujo número de massa $\mathrm{A}=\mathrm{Z}+\mathrm{N}$, em que $\mathrm{N}=$ número de nêutrons. Daí sua relação com número atômico Z (7.15.1). ${ }^{29}$

Em 1920, Rutherford sugeriu que elétrons poderiam estar ligados a prótons sem ter existência independente no núcleo. A essa partícula, deu o nome de nêutron. Porém para detectá-lo houve dificuldade, por não ter carga e não ser desviado por campo magnético nem ionizar campos elétricos.

28 A massa atômica de todos os elementos é aproximadamente múltipla da massa do hidrogênio. O procedimento de Rutherford foi proposital, pois ao bombardear um elemento com número atômico $Z=n \operatorname{com}$ partículas $\alpha$, de $Z=2$, se obtém um isótopo instável de $Z=n+2$, que decai para um isótopo de $Z=n+1$ mais um próton ${ }_{1} H^{1}$ de $Z=1$, de acordo com a hipótese de Prout. Ver Observação adiante, em 7.15.1.

${ }^{29}$ Quando Prout emitiu sua hipótese, não se sabia da existência de prótons e nêutrons no núcleo do átomo. Devemos explicar a diferença entre o número de massa $A$ e massa atômica: foi escolhido o Carbono $12, C^{12}$ como padrão com número de massa 12, que passou a ter 12 uma, unidade de massa atômica, e os outros elementos são comparados com a massa do $C^{12}$. O número de massa é a soma número de prótons com o número de nêutrons. Por exemplo: o número de massa do cloro é 35 , mas a massa atômica é 35,453. Por que a massa atômica é fracionária? Os elementos são formados por uma mistura de isótopos. No caso do cloro, $75,77 \%$ dos átomos têm número de massa 35, 24,23\%, têm número de massa 37 e uma quantidade muito pequena tem $\mathrm{n}^{\circ}$ de massa diferente de 35 e 37 . A massa atômica é a média ponderada desses isótopos. Se examinarmos as reações atômicas do Exemplo ilustrativo da Seção 7.8.1, sobre a fissão e a fusão nuclear, verificamos que há uma diferença entre antes e depois da reação, que somente foi explicado pela fórmula: $E=c^{2} \Delta m$, a transformação de massa em energia. 
Finalmente, em 1932, como resultado da pesquisa da desintegração ou transmutação de núcleos por partículas $\alpha$, Chadwick demonstrou sua existência, o que conduziu à constituição do núcleo com prótons e nêutrons ver reação $C^{13} \alpha, n O^{16} \quad(0)$.

Mais recentemente, em 1964, Murray Gell-Mann e George Zweig, independentemente, sugeriram que prótons e nêutrons, classificados como bárions, assim como os mésons, fossem constituídos por subunidades, denominadas por Gell-Mann como quarks. O quark down foi descoberto em 1980 e o quark up, em 1996 no acelerador de partículas Tevatron, do Fermilab.

Prótons e nêutrons seriam formados por quark up, símbolo u, e quark down, símbolo d, da seguinte forma:

Próton formado por uud, com a carga: $(+2 / 3)+(+2 / 3)+(-1 / 3)=+1$

Nêutron formado por udd, com a carga: $(+2 / 3)+(-1 / 3)+(-1 / 3)=0$. 